\title{
Paired Subimage Matching Watermarking Method on Ordered Dither Images and Its High-Quality Progressive Coding
}

\author{
Jing-Ming Guo, Member, IEEE, Soo-Chang Pei, Fellow, IEEE, and Hua Lee, Fellow, IEEE
}

\begin{abstract}
In this paper, we present two novel robust methods for embedding watermarks into dithered halftone images. The first method is named Paired Subimage Matching Ordered Dithering (PSMOD), of which the decoder is provided with a priori information of the original watermark, and the corresponding application is copyright protection. The other method, Blind Paired Subimage Matching Ordered Dithering (BPSMOD), does not require the knowledge of the original watermark, and the main application is secret communication. Both methods utilize the bit and sub-subimage interleaving preprocesses. The experiments show that both techniques are sufficiently robust to guard against the cropping, tampering, and print-and-scan degradation processes, in either B/W or color dithered images. Both techniques are also sufficiently flexible for various levels of embedded capacities. Furthermore, a novel progressive coding scheme is also presented in this paper for the efficient display of dithered images. After the preprocessing of bit-interleaving, this algorithm utilizes the characteristic of reordered image to determine the transmitting order and then progressively reconstructs the dithered image. Moreover, the dithered images are further compressed by lossy and lossless procedures. The experimental results demonstrate high-quality reconstructions while maintaining low transmitted bit rates.
\end{abstract}

Index Terms-Bit-interleaving, error diffusion, halftone, ordered dither, watermarking.

\section{INTRODUCTION}

D IGITAL halftoning is to convert multitone images into two-tone format, which is commonly used in computer printouts, books, newspapers, and magazines [1], [2]. When viewed at a proper distance, halftone images resemble the original due to the lowpass nature of the human visual systems. In practice, two most common types of halftoning techniques are ordered dithering and error diffusion [3]-[5]. Between these two, ordered dithering is the most efficient and offers good visual quality.

Manuscript received July 2, 2006; revised September 10, 2007. This work was supported by the National Science Council of Taiwan, R.O.C., under Contract 96-2221-E-011-124-MY2. The associate editor coordinating the review of this manuscript and approving it for publication was Dr. Bo Shen.

J.-M. Guo is with the Department of Electrical Engineering, National Taiwan University of Science and Technology, Taipei, Taiwan, R.O.C. (e-mail: jmguo@seed.net.tw).

S.-C. Pei is with the Department of Electrical Engineering, National Taiwan University, Taipei, Taiwan, R.O.C. (e-mail: pei@cc.ee.ntu.edu.tw).

H. Lee is with Department of Electrical and Computer Engineering, University of California, Santa Barbara, CA 93106-9560 USA (e-mail: hualee@ece. ucsb.edu).

Color versions of one or more of the figures in this paper are available online at http://ieeexplore.ieee.org.

Digital Object Identifier 10.1109/TMM.2007.911259
Several methods for watermarking in halftone images have also been developed. These techniques are regularly applied to the printing of security documents such as ID card, currencies, and confidential documents, to prevent illegal duplication and forgery. These methods can be divided into three categories.

The first category involves low computational complexity using ordered dithering, but non-flexible embedded capacity watermarking. The method uses different dither cells to create a threshold pattern in the halftoning process with each dither cell representing the corresponding information bit in the watermark [6], [7]. One another approach employs pixel-wised data hiding by smart pair toggling (DHSPT), achieves excellent quality by choosing the minimum connection toggled pixels after data embedding [8].

The second category has higher computational complexity for direct binary search and error diffusion. These methods are capable of obtaining higher quality embedded image watermarking, which includes obtaining good visual quality as well as good watermark detection with joined direct binary search (DBS) halftoning and spread spectrum watermarking algorithms [9]. There are some applications of vector quantization (VQ) to embed watermarks into the most or least significant bit (MSB/LSB) of error diffusion images. The benefits of this approach are that a low bit-depth halftone for printing or progressive transmission can be achieved by masking one or more bits off from the higher bit-depth image [10], [11]. Adopting modified data hiding error diffusion (MDHED) to embed data into error diffusion images, where the method is effectively to hide a relative amount of data with good quality halftone images, and the amount of hidden data is easy to control [12]. Using constructed LUT pairs to generate the state sequence, and then embeds the corresponding compressed version along with watermark and LUT into blocks that meets some predefined constraints. In decoder, the original image can be recovered using the reverse process of watermark embedding [13].

The third category has low computational complexity. However, the decoded result is an approximation of the original watermark. This is to embed a watermark into the dithered images by using a pair of conjugate halftone screens. Fourier analysis can be utilized to determine the periodicity of the embedded print-and-scan image, and then the embedded watermark can be obtained by the superposition of correlated portions of the image [14]. Embedding hidden visual patterns into two or more halftone images, the hidden visual patterns can be perceived directly when the halftone images are overlaid each other. These techniques include using stochastic screen patterns [15], sto- 


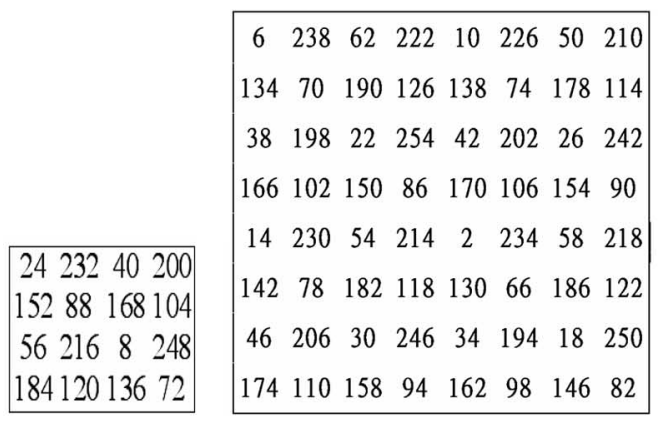

(a)

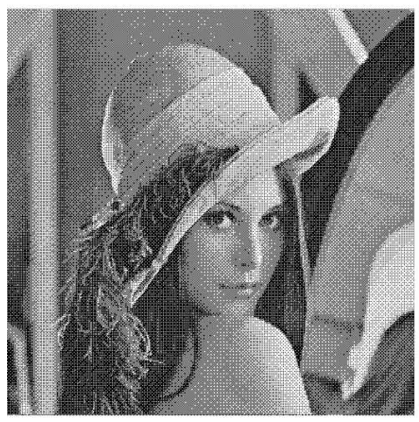

(e) (b)

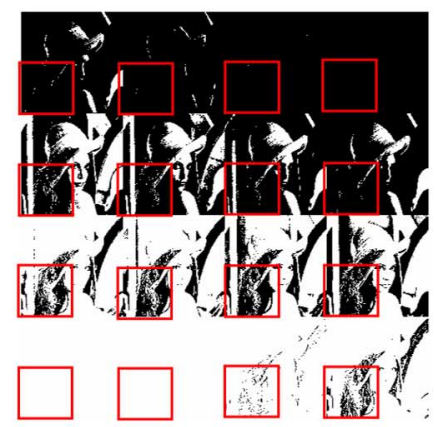

(f)

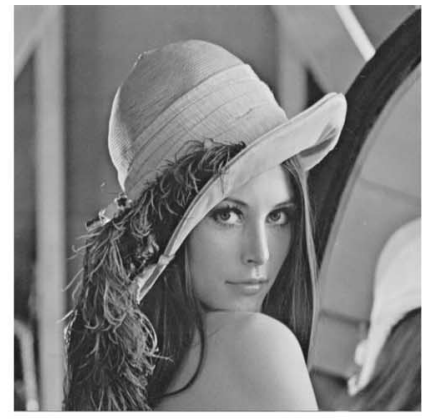

(c)

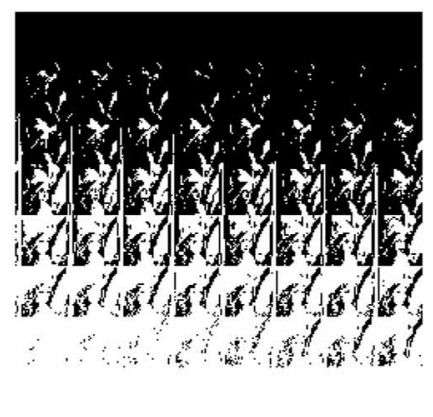

(g)

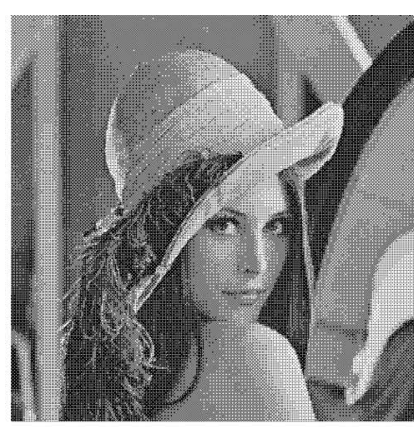

(d)

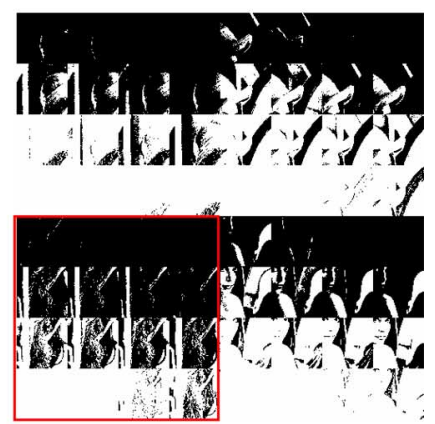

(h)

Fig. 1. (a) $4 \times 4$ dispersed halftone screen; (b) $8 \times 8$ dispersed halftone screen; (c) Original $512 \times 512$ grayscale Lena image. (d) Dithered image obtained by $4 \times 4$ halftone screen (PSNR $=32.29 \mathrm{~dB})$. (e) Dithered image obtained by $8 \times 8$ halftone screen $(\mathrm{PSNR}=32.52 \mathrm{~dB})$. (f) Bit-interleaving result from $1(\mathrm{c})$. $(\mathrm{g})$ Bit-interleaving result from 1(e). (h) After image dividing and SSI-interleaving processes from 1(f) (all printed at 300 dpi).

chastic error diffusion [16], conjugate error diffusion [17], conjugate error diffusion in color halftone images [18], noise-balanced error diffusion [19], and hybrid pixel-based data hiding and block-based watermarking [20].

As for image compression, consider the case that a user is to select one from a number of images in a remote database. Assume the images are sequentially compressed prior to transmission. The lossless compression required gives an average compression ratio ranging from 1 to 2 . In narrow bandwidth wireless environment, the compression ratio will not be practical. So, a more effective approach is to use progressive coding to transmit an approximation of the images first at low bit rates. If the user identifies the image of interest, further refinement can be requested. For this, Kollias and Anastassiou proposed a progressive coding scheme for error-diffused halftone images using a distortion criterion [21].

In this paper, the ordered dither halftone image is the main image format. One interesting "ramp structure" after bit-interleaving [22] or sub-subimage-interleaving is observed in Fig. 1. This phenomenon is quite straight forward to be used in watermarking and progressive coding applications according to the proposed coding approaches. In watermarking, we elaborate the three different categories. Among these, the proposed approach belongs to the first one. However, in this category, the methods in [6] and [7] do not have the flexibility for various embedded capacities. Moreover, according to the experiments introduced later, those image qualities are inferior to the proposed method as well as the one obtained by Fu and Au's method [8]. In general, Fu and Au's method achieves the best image quality. However, in terms of robustness, it is not able to withstand the harm from distortions. For these, we propose two robust watermarking techniques by embedding watermark into a dithered image after the pre-process of bit-interleaving and subimage-interleaving. These methods are capable of achieving good visual quality and flexibility for various embedded capacities, while maintaining low computational complexity of ordered dithering using threshold process. In progressive coding, the algorithm proposed by Kollias and Anastassiou [20] can only be used in error-diffused halftone images. However, the subject of this work is on ordered dither halftone image. Hence, we develop a novel progressive coding scheme for dithered images. The performance of the algorithm is measured by the PSNR that cooperates with the human visual system between the original dithered image and the reconstructed dithered image at every step. Experimental results show quality halftone reconstruction and high compression ratios can be achieved.

The rest of the article is organized as follows. Bit-interleaving, which is to bridge the components of watermarking and progressive coding, is introduced in Section II. Section III describes the error criterion. Section IV outlines the proposed watermarking in dithered images and Section V is the proposed progressive coding approach. The experimental results are documented in Section VI, and Section VII draws conclusions.

\section{BIT-INTERLEAVING}

Halftoning screens are designed for various specific purposes. As an example, Fig. 1(a) and (b) show two dispersed-dot dithering screen [1]. Generally speaking, the choice of dispersed-dot screen and clustered dot screen is mainly based on printing process problems. Clustered dot screens can hide 

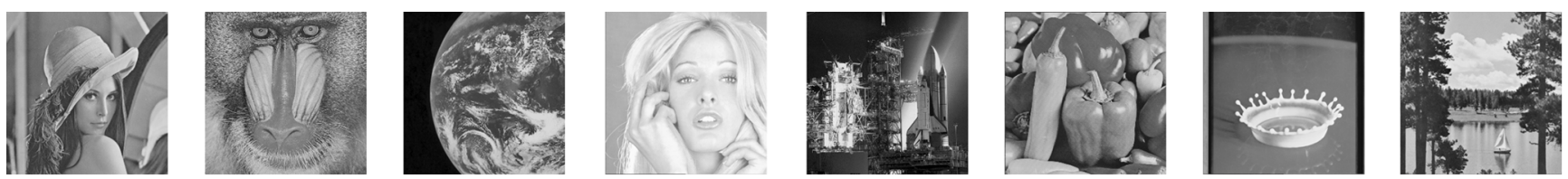

Fig. 2. Thumbnail of 8 tested images. Left to right: Lena, Mandrill, Earth, Tiffany, Shuttle, Peppers, Milk, and Lake.

artifacts commonly seen in laser printing, such as dot gain effect. But the image quality is lower because of the lack of spatial resolution. On the other hand, dispersed dot screens provide higher image quality. However, they exacerbate process artifacts of laser printing and are therefore mainly used in inkjet printers. More threshold elements in a dithered screen render more color, but they also decrease the spatial resolution as well. To illustrate the algorithm of ordered dithering, we define the halftone screen as $M \times N$ matrix array. Each pixel $x_{i, j}$ in the original gray level image is mapped to a halftone screen value $H S_{i \bmod M, j \bmod N}$. The dithered output $b_{i, j}$ is determined as

$$
b_{i, j}=\left\{\begin{array}{ll}
255(\text { white }), & \text { if } x_{i, j} \geq H S_{i \bmod M, j \bmod N} \\
0(\text { black }), & \text { if } x_{i, j}<H S_{i \bmod M, j \bmod N}
\end{array} .\right.
$$

Fig. 1(d) and (e) are the dithered Lena images of the original grayscale image, shown as Fig. 1(c), processed with halftone screen given as Fig. 1(a) and (b). The bit-interleaving procedure extracts and gathers all the pixels corresponding to the same threshold value in Fig. 1(a) and (b) to form the final image, shown as Fig. 1(f) and (g). In this case, the final images are composed of 16 and 64 subimages, respectively.

\section{ERROR CRITERION}

Let the size of the original image be $P \times Q$. The error criterion is defined as

$$
\mathrm{PSNR}=10 \log _{10} \frac{P \times Q \times 255^{2}}{\sum_{i=1}^{P} \sum_{j=1}^{Q}\left[g_{i, j}-\sum_{u, v \in R} \sum w_{u, v} b_{i+u, j+v}\right]^{2}}
$$

where the variables $g_{i, j}$ and $b_{i, j}$ represent the original gray level image and the corresponding halftone images in position $(\mathrm{i}, \mathrm{j})$, respectively; $R$ is the support region of the human visual coefficients and $w_{u, v}$ is the human visual coefficient at $(u, v)$, which can be obtained by psychophysical experiments [23], [24]. The other method to obtain $w$ is to use a training set of both pairs of gray level images and good halftone results of them, such as using error diffusion or ordered dithering to produce the set. Here we use Least-Mean-Square (LMS) to derive $w$ as described as follows:

$$
\begin{aligned}
& \hat{g}_{i, j}=\sum_{u, v \in R} \sum w_{u, v} b_{i+u, j+v}, \\
& e_{i, j}^{2}=\left(g_{i, j}-\hat{g}_{i, j}\right)^{2}, \\
& \frac{\partial e_{i, j}^{2}}{\partial w_{u, v}}=-2 e_{i, j} b_{i+u, j+v},
\end{aligned}
$$

$\left\{\right.$ if $w_{u, v}>w_{u, v, \text { opt }}$, slope $>0, w_{u, v}$ should be decreased $\left\{\right.$ if $w_{u, v}<w_{u, v, \text { opt }}$, slope $<0, w_{u, v}$ should be increased

$w_{u, v}^{(k+1)}=w_{u, v}^{k}+\mu e_{i, j} b_{i+u, j+v}$ where

$$
\begin{array}{ll}
\hat{g}_{i, j} & \text { reconstructed gray level pixel at position }(\mathrm{i}, \mathrm{j}) ; \\
R & \begin{array}{l}
\text { support region of the LMS filter (e.g., } 5 \times 5 \text { or } \\
\end{array} \\
w_{i, j} & \text { LMS coefficient at position (i,j); } \\
w_{i, j, o p t} & \text { optimum LMS coefficient; } \\
e_{i, j}^{2} & \text { MSE between } g_{i, j} \text { and } \hat{g}_{i, j} ; \\
\mu & \text { adjusting parameter used to control the convergent } \\
& \text { speed of the LMS optimum procedure, with the } \\
& \text { value set to } 10^{-5} \text { in this paper. Next paragraph we } \\
& \text { give a series of experiments to demonstrate the } \\
& \text { selection of this value. }
\end{array}
$$

Following we conducted extensive experiments to obtain the optimum size of LMS filter and the value of parameter $\mu$. There are 8 training images as shown in Fig. 2 involved in our training process: Lena, Mandrill, Peppers, Milk, Shuttle, Earth, Lake, and Tiffany images. Three types of different original halftone techniques, including error diffusion, dispersed-dot dithering, and clustered-dot dithering, are employed here. Four different LMS filter sizes, $3 \times 3,5 \times 5,7 \times 7,9 \times 9$, and three different value of parameter $\mu$ of $10^{-4}, 10^{-5}, 10^{-6}$ are also tested in this study. The convergent speed is depicted in Fig. 3. The vertical axis stands for the absolute overall changes of the LMS filter in each training iteration and is defined as following:

$$
\text { change }(k)=a b s\left(\sum_{u, v \in R} \sum w_{u, v}^{(k)}-w_{u, v}^{(k-1)}\right),
$$

where $\mathrm{k}$ represents the number of iteration. The smaller the value of change $(\mathrm{k})$ is, the closer it approximates the convergence. In Fig. 3, it is clear that the parameter $\mu$ with value $10^{-6}$ takes copious time to converge, which also gets longer as the filter size grows up. On the other hand, the parameter $\mu$ with value $10^{-5}$ gives reasonable convergent speed, usually within 5 iterations, and the parameter $\mu$ with value $10^{-4}$ achieves highest convergent speed. However, it cannot give superior reconstructed halftone quality to the filter obtained from parameter $\mu$ with value $10^{-5}$ and $10^{-6}$, which will be discussed in the following experiment. In Fig. 4, we give reconstructed mandrill halftone results with different combination of LMS filter size and parameter $\mu$. Note that the average PSNR under each reconstructed halftone images stands for the average PSNR value of 8 test images described above. In Fig. 4, it is clear that the LMS filter size with $3 \times 3$ leads to artifacts similar to false contour in the reconstructed halftone images. On the other hand, filter sizes of $9 \times 9$ or larger only offer little benefit in quality, but substantially increase the computational 


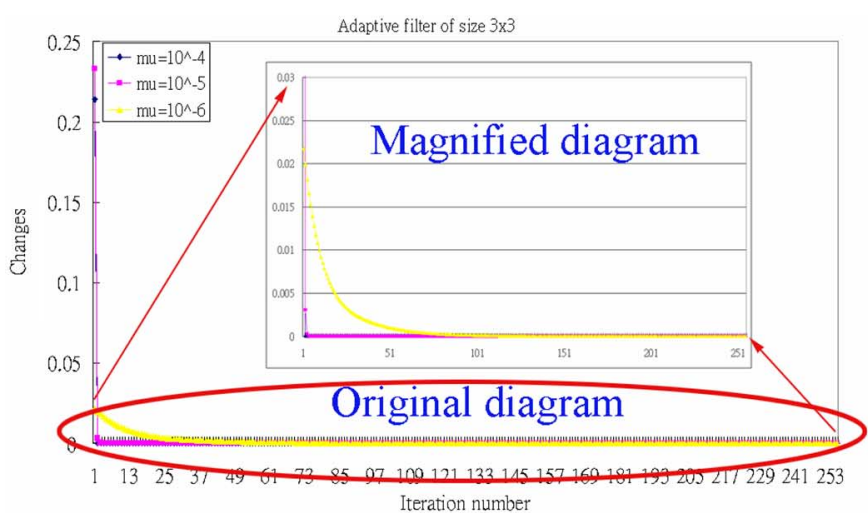

(a)

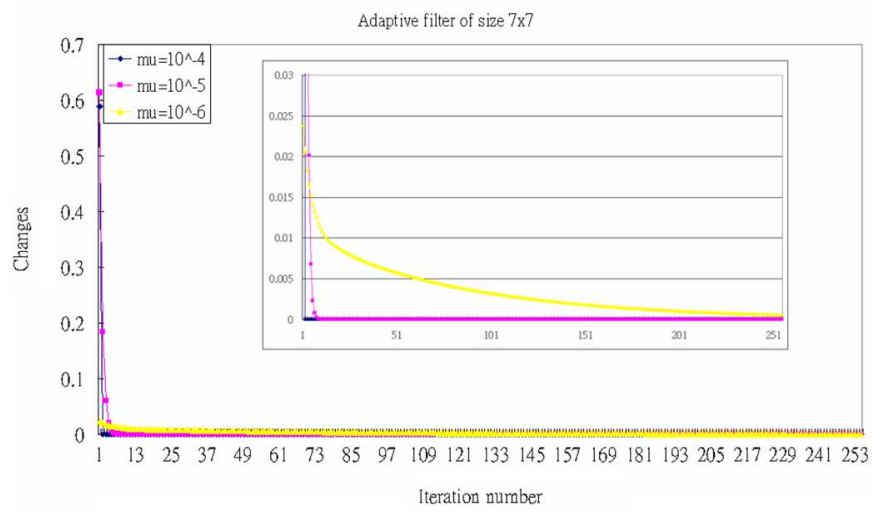

(c)

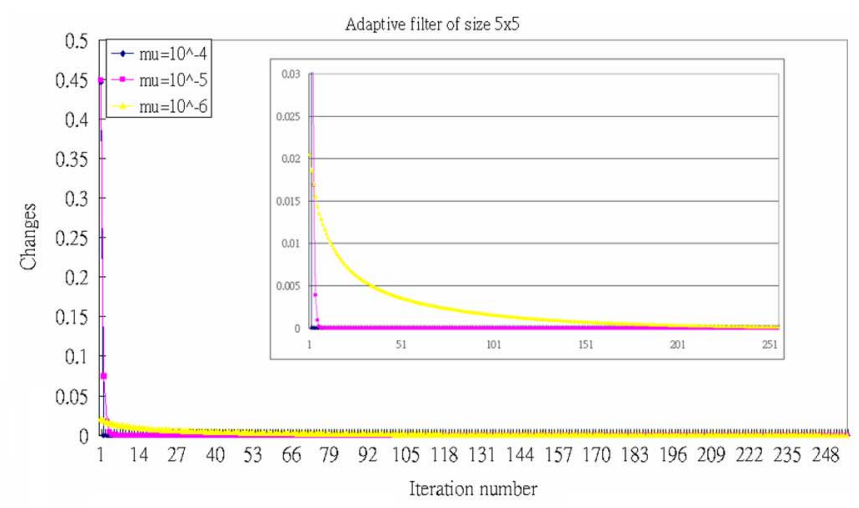

(b)

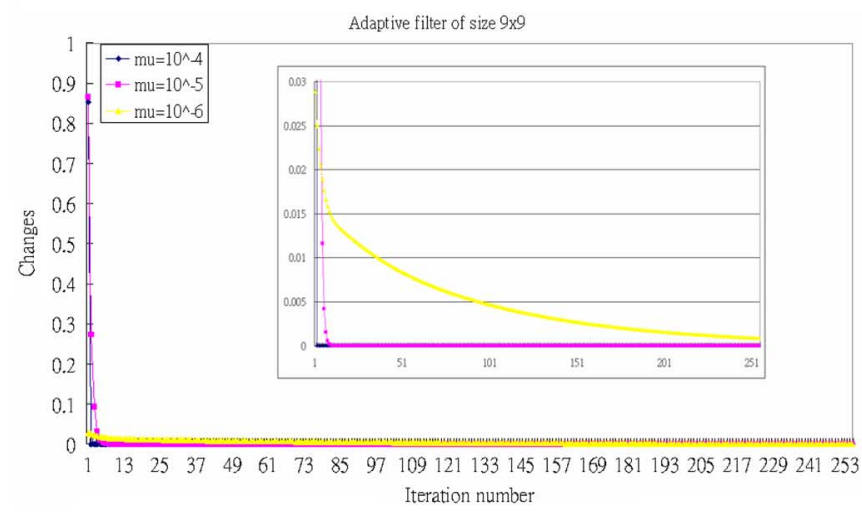

(d)

Fig. 3. Convergent speed under different size of LMS adaptive filter and parameter $\mu$. (a) $3 \times 3$. (b) $5 \times 5$. (c) $7 \times 7$. (d) $9 \times 9$.

complexity. Therefore, the filter sizes of $5 \times 5$ or $7 \times 7$ are more suitable for reconstructing the halftone images. Among them, the filter of the size $7 \times 7$ generally gives higher halftone quality than $5 \times 5$, although it has higher computational complexity. So, in this paper, we select filter size $7 \times 7$, as shown in Fig. 5, for objective quality evaluation. In Fig. 4, we also find that in different value of parameter $\mu$, the LMS filter of value $10^{-5}$ achieves comparable reconstructed halftone quality at the value of $10^{-6}$. In Summary, the parameter $\mu$ with value $10^{-5}$ gives reasonable convergent speed and good reconstructed halftone quality, so in this paper we employ this value for the LMS filter coefficients evaluation.

In this paper we adopt the LMS filter of the size $7 \times 7$ as the quality criterion. Notice that the filter has the basic characteristic of human visual system:

1) The diagonal $\left(45^{\circ}\right)$ has less sensitivity (lower values) than vertical and horizontal directions.

2) The center has the highest sensitivity and decay with the increasing distance to center.

With this criterion, the quality of the original dithered images shown in Fig. 1(d) and (e) are measured at 32.29 and 32.52 dB, respectively.

\section{WATERMARKING IN DITHERED IMAGES}

Two cases often occur in practical applications. One is that the receiver is provided with a priori information of the watermark. For this case, the Paired Subimage Matching Ordered Dithering (PSMOD) method is suitable for encoding. One direct application of PSMOD is copyright protection. The other is when the knowledge of the watermark is not available, and the Blind Paired Subimage Matching Ordered Dithering (BPSMOD) method can be applied. The main application of BPSMOD is secret communication. Fig. 6 is the flow chart of the proposed watermarking techniques. The details of the techniques are described as follows.

\section{A. Paired Subimage Matching Ordered Dithering (PSMOD)}

Let $x_{i, j, 1}, x_{i, j, 2}, \ldots \ldots, x_{i, j, m}$ be the subimage sequence after bit-interleaving. The three scripts, $i, j$, and $m$ specify pixel $(\mathrm{i}, \mathrm{j})$ from subimage $\mathrm{m}$. Suppose the image is of size $P \times Q$, and is divided into $\mathrm{M}$ and $\mathrm{N}$ subimages along vertical and horizontal orientations, respectively. The range of $\mathrm{i}=0,1, \ldots \mathrm{P} / \mathrm{M}$, and the range of $\mathrm{j}=0,1, \ldots \mathrm{Q} / \mathrm{N}$. Suppose $\mathrm{m}=n^{2}$, we pick $\mathrm{m}=64$ for $\mathrm{n}=8$ for this example. From the left to right, bottom to top, we pair the subimages as $\left(x_{i, j, k}, x_{i, j, k+1}\right), k=2 \mathrm{t}+1$, for $\mathrm{t}=0,1, \ldots, 31$, to produce 32 pairs of subimages. For each pair, we assume $x_{i, j, k+1}$ has more black pixels than $x_{i, j, k}$, which is a reasonable assumption. This is because the left subimage corresponds to smaller value of threshold in halftone screen than the right subimage. So the left subimage is more likely to produce more white pixels than the right subimage. We then define $x_{i, j, k+1}$ as the black subimage and $x_{i, j, k}$ as the white subimage. The original order $\left(x_{i, j, k}, x_{i, j, k+1}\right)$ represents a white pixel in watermark and $\left(x_{i, j, k+1}, x_{i, j, k}\right)$ represents a black pixel. In the decoder, the watermark is extracted by an AND operation between 


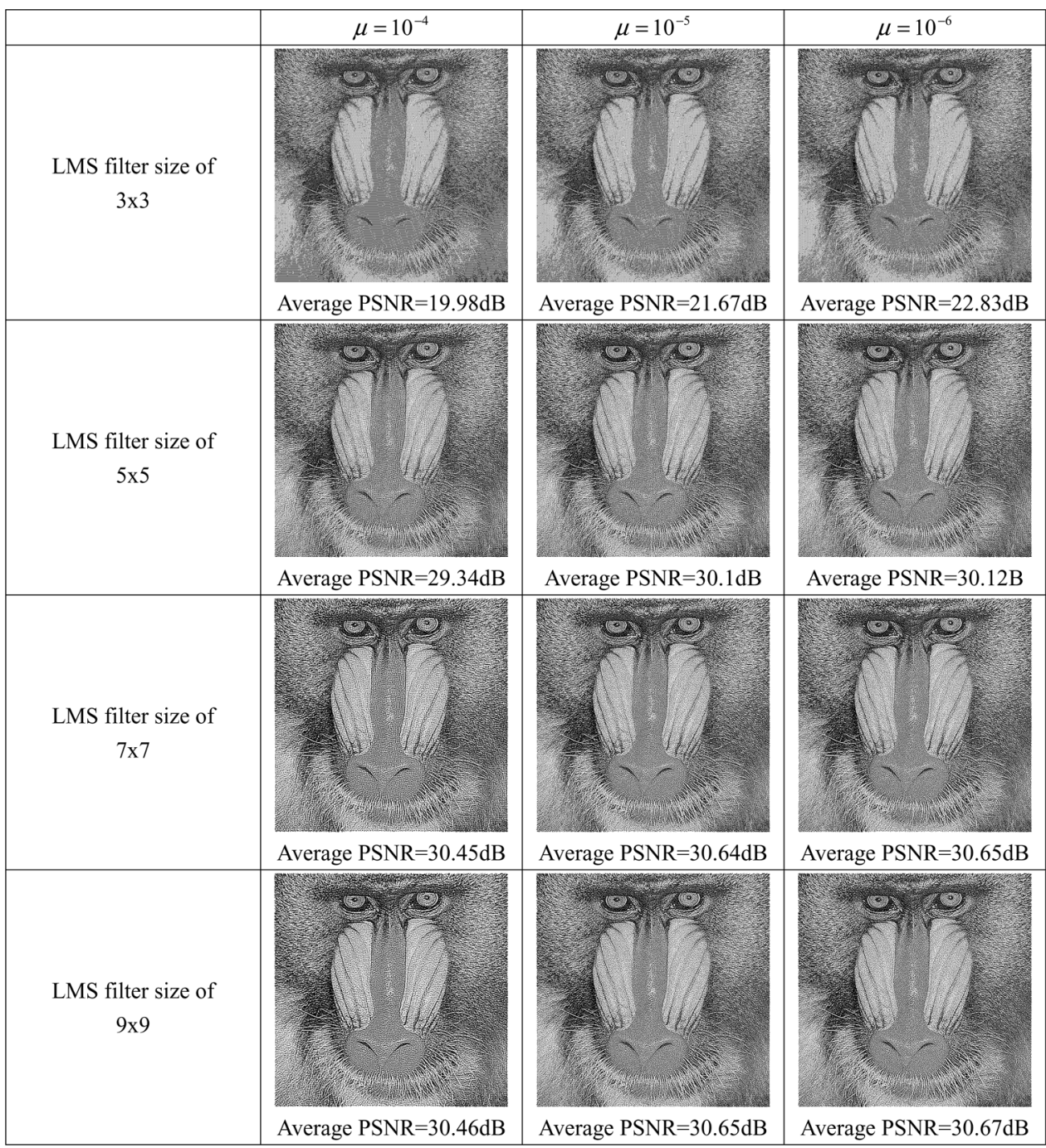

Fig. 4. Reconstructed halftone image quality under different LMS adaptive filter and parameter $\mu$.

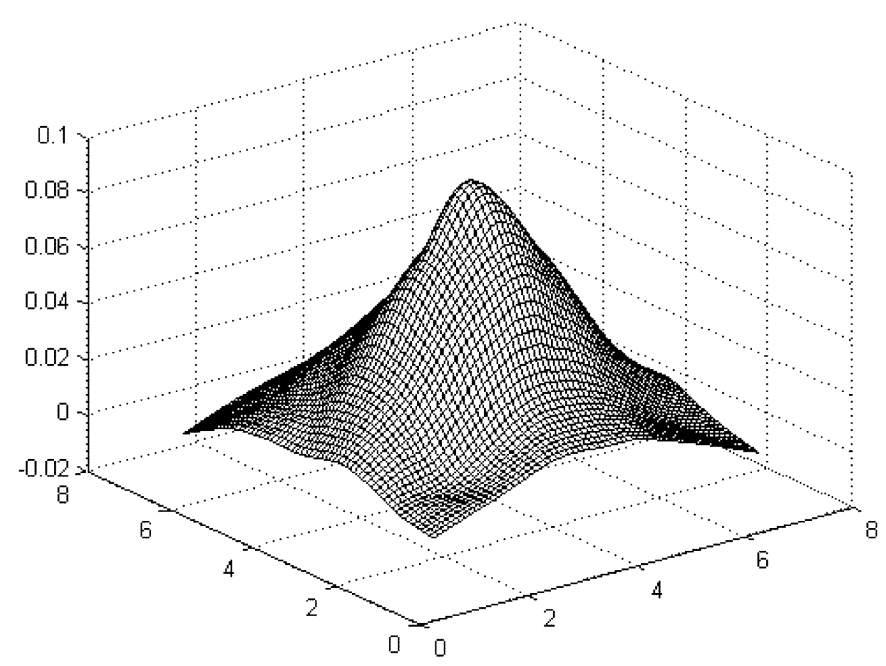

Fig. 5. LMS-trained human visual filter $(7 \times 7)$.

the watermarked image and the original watermark. Here, the white pixel is taken as logic-0, and black pixel as logic-1 during the AND decoding procedure. Since the watermark is extracted by the AND operation, it is necessary to eliminate the
Non-Increased black pixel Pairs (NIP) in the relative position only where the watermark has a black pixel.

Subsequently, we focus on the improvement of the embedded capacity by the PSMOD method. First, we enlarge the halftone screen from $8 \times 8$ to $16 \times 16$, so that, after bit-interleaving, 128 bits can be used for embedding. In the watermarking scheme, the accurate decoding result is based on the assumption that, in the pair of subimages $\left(x_{i, j, k}, x_{i, j, k+1}\right), x_{i, j, k+1}$ contains more black pixels than $x_{i, j, k}$. If the likelihood of $x_{i, j, k}$ has more black pixels than $x_{i, j, k+1}$, the erroneous decoding rate increases as well. The decoding error rate (erroneous error rate) is obtained by pseudo random generating 100 different watermarks and then embedded into image without pseudo pixels using PSMOD. Since there is no pseudo pixel used to eliminate the NIPs, the decoded watermarks may not be the same as the original ones. The decoding error rate is defined as the average hamming distance between the decoded watermarks and the original watermarks divided by the size of one watermark. The statistics of NIP is shown in Table I. For the experiments, eight images, as described in Section III, are utilized for testing. Table I indicates that the erroneous decoding rate of every embedded bit increases rapidly from $9.1 \%$ to $21.7 \%$ as the size of halftone screen increases from $8 \times 8$ to $16 \times 16$. 


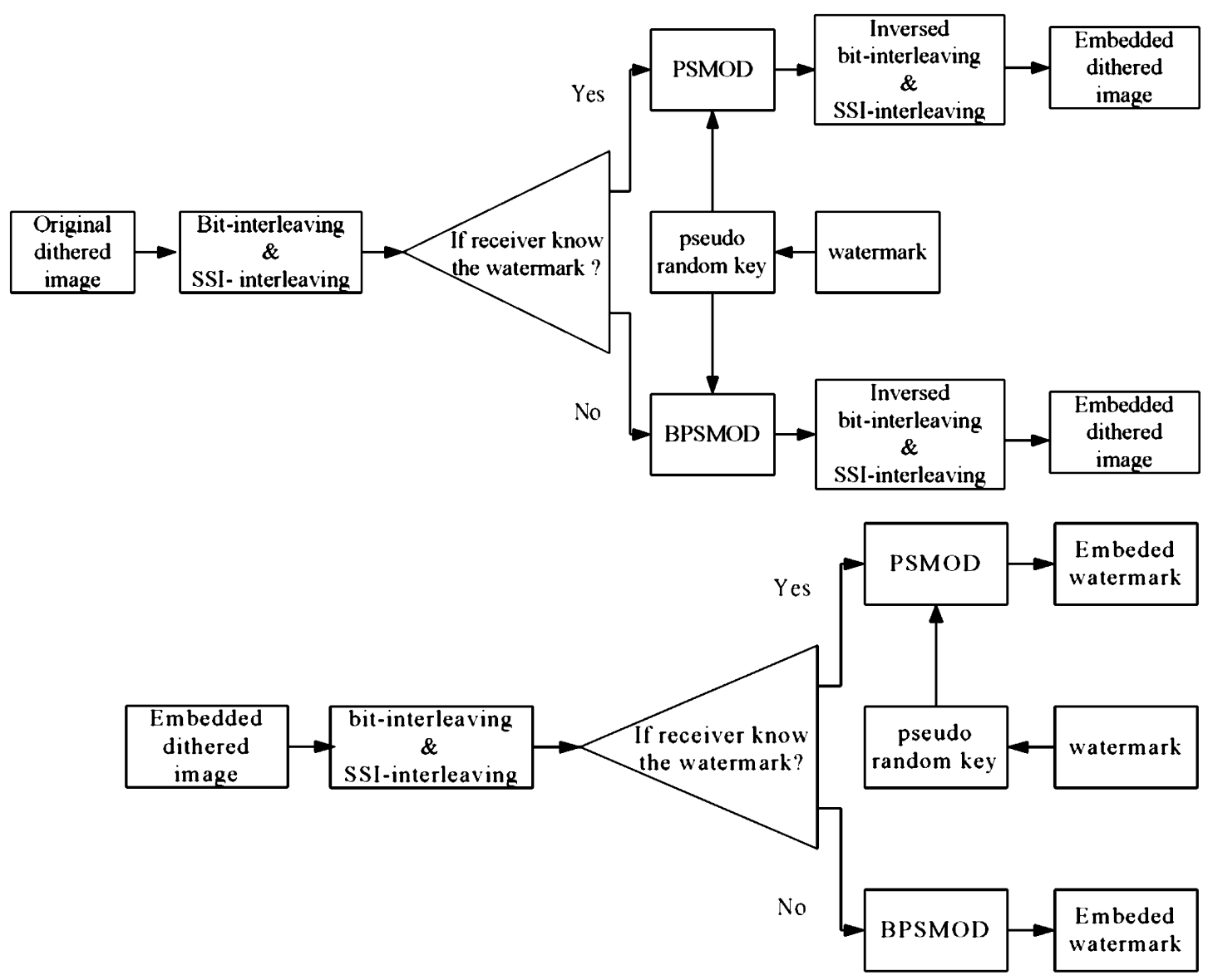

Fig. 6. Flow chart of the proposed watermarking scheme (upper: encoder, bottom: decoder).

TABLE I

Statistical Number of Non-Increased Black Pixel Pairs (NIP), Additive Pseudo Pixels, and Decoding Error Rates

\begin{tabular}{|l|c|c|c|c|c|c|c|c|}
\hline Size of halftone screen & $4 \times 4$ & $4 \times 4$ & $4 \times 4$ & $4 \times 4$ & $8 \times 8$ & $8 \times 8$ & $8 \times 8$ & $16 \times 16$ \\
\hline Dividing number of every sub-image & 1 & 4 & 16 & 64 & 1 & 4 & 16 & 1 \\
\hline embedded watermark bits & 8 & 32 & 128 & 512 & 32 & 128 & 512 & 128 \\
\hline Average number of NIP & 1 & 5 & 35 & 230 & 6 & 38 & 254 & 56 \\
\hline Number of additive pseudo pixels & 1 & 18 & 68 & 327 & 21 & 130 & 575 & 320 \\
\hline Decoding error rate (\%)(without pseudo pixels) & 5.3 & 7.8 & 13.7 & 22.5 & 9.1 & 15 & 24.8 & 21.7 \\
\hline PSNR (dB) & 31.35 & 31.33 & 31.24 & 30.79 & 30.59 & 30.41 & 29.58 & 30.2 \\
\hline
\end{tabular}

The second method utilizes the strategy of further dividing each bit-interleaved subimage into "sub-subimage (SSI)" and then executes the "SSI-interleaving" over again. An example of the image dividing and SSI-interleaving process is shown in Fig. 1. In Fig. 1(f), each subimage is further divided into 4 SSI's, and then reorder the 16 SSI's marked with the red squares in Fig. 1(f) into the large red square in Fig. 1(h). If we pair the SSI's in Fig. 1(h) into $\left(x_{i, j, k}^{\prime}, x_{i, j, k+1}^{\prime}\right)$, where $\mathrm{k}=2 \mathrm{t}+1$ and $\mathrm{t}=$ $0,1, \ldots, 31$ as before, $x_{i, j, k+1}^{\prime}$ still normally has more black pixels than $x_{i, j, k}^{\prime}$, In this way, we still can embed a watermark of 32 bits although the size of halftone screen is fixed at $4 \times 4$.

Some observations are noteworthy.

1) For fixed halftone screen size, the size of embedded watermark can be increased by image dividing and SSI-inter- leaving. The erroneous decoding rate increases slower than the direct use of larger halftone screen size.

2) Smaller of halftone screen size and more divided SSI's result in lower decoding error rate. However, a smaller halftone screen renders fewer gray levels of dithered image at a viewing distance.

Finally, we examine the solution to the NIP problem. The NIP problem can be solved if we adequately increase the number of black pixels in $x_{i, j, k+1}\left(x_{i, j, k+1}^{\prime}\right)$ subimage or increase the number of the white pixels in $x_{i, j, k}\left(x_{i, j, k}^{\prime}\right)$. We name the additive black and white pixels as pseudo-pixels. Because of the low-pass characteristics of the human visual system (HVS), the best additive pseudo-pixels position is in the high-frequency region of an SSI. When an NIP occurs, we use the concept of 


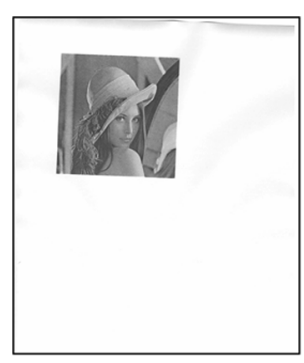

(a)

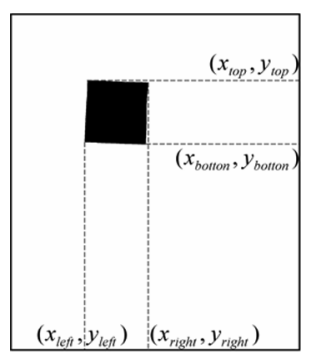

(f)

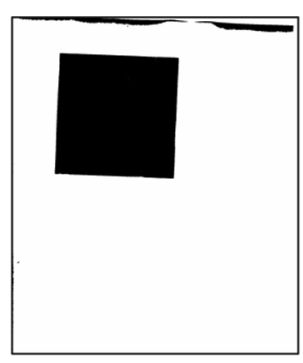

(b)

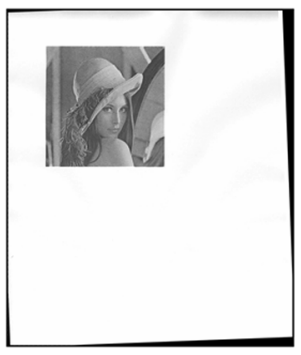

(g)

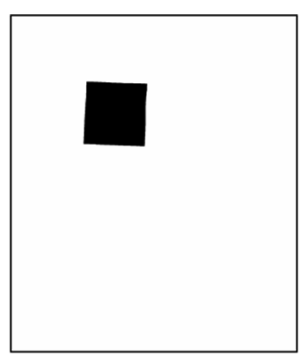

(c)

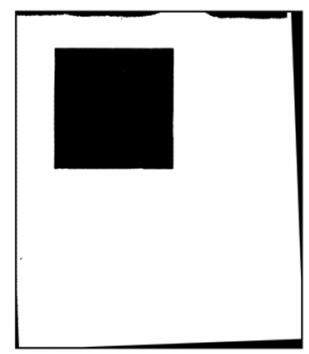

(h)

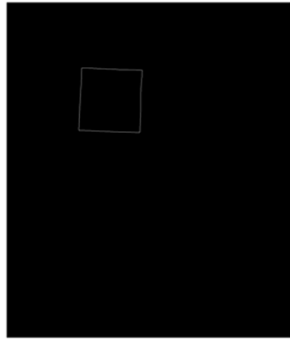

(d)

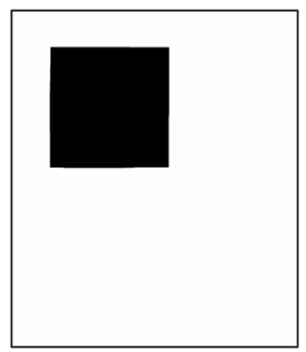

(i)

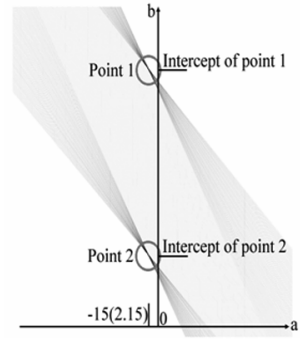

(e)

Fig. 7. Proposed recovery procedure for print-and-scan attack. (a) Lowpass filtered result. (b) Binarized result. (c) Morphological expansion and erosion processed result. (d) Edge detected result. (e) Hough transform in a-b domain. (f) Coordinates of the projected boundaries. (g) Rotation corrected result. (h) Corrected result of the mask image. (i) Halftone image localization mask. (j) Image size normalized result. (k) Normalized image binarization. (a) $f^{(1)}(i, j),\left(\right.$ b) $f^{(2)}(i, j)$, (c) $f^{(3)}(i, j)$, (d) $f^{(4)}(i, j)$, (e) (f) (g) $f^{(5)}(i, j)$, (h) $f^{(6)}(i, j)$, (i) $f^{(7)}(i, j),(\mathrm{j}) f^{(8)}(i, j)$, and (k) $f^{(9)}(i, j)$.

sliding window, which only covers two pixels in an SSI. If the values of the two pixels covered by the sliding window are different, it implies a high frequency region in this window. Suppose the black pixel number should be decreased in this SSI, the black pixel in the window is flipped to white, and vice versa. If the pair of SSI's are both in whole black or whole white, that means wherever we move the sliding window inside either of the two SSI, the pixel values are always the same (always low frequency region). In this case, we can place the pseudo-pixel in any position of either of the two SSI to eliminate the NIP. The average additive pseudo-pixels number used to solve NIP problem in eight tested images is shown in Table I. For the same size of embedded watermark, the dithered image with more dividing requires fewer pseudo-pixels.

\section{B. Blind Paired Subimage Matching Ordered Dithering (BPSMOD)}

In secret communication application, the receiver is not given the information of the original watermark. For these cases, the PSMOD method will fail. The basic difference between PSMOD and BPSMOD is that, in PSMOD, the NIP is necessary to be eliminated only in the relative position where the watermark has a black pixel. However, in BPSMOD, all of the NIP should be eliminated. So in the decoder the original order $\left(x_{i, j, k}^{\prime}, x_{i, j, k+1}^{\prime}\right)$ represents a white pixel in watermark and $\left(x_{i, j, k+1}^{\prime}, x_{i, j, k}^{\prime}\right)$ represents a black pixel. The procedures within the receiver, which includes bit-interleaving, subimage dividing, and SSI-interleaving are practically the same as the PSMOD.

\section{Watermark Extraction After Print-and-Scan Process}

In the most common applications of halftoning in printing books, newspapers, and magazines, the original embedded watermarked image is often destroyed by the print-and-scan process, e.g., zooming, rotation, and dot gain. Extracting the original watermarks perfectly is a difficult task. For this, we propose an automatic procedure for resynchronization after print-and-scan attack as introduced below.

Step 1) Lowpass filtering: From this stage to step 5, we try to locate the halftone image from the printed document and solve the rotation issue inherent in print-andscan attacked image. Since black and white pixels generally appear alternately in halftone image, the lowpass filtering is to make the region inside the halftone image as a cluster of dark area. Suppose the image is of size $P \times Q$, the lowpass filtering is defined as below. A filtered example is shown in Fig. 7(a).

$$
\begin{aligned}
& f^{(1)}(i, j)=\sum_{m=-1}^{1} \sum_{n=-1}^{1} \frac{f(i+m, j+n)}{9}, \\
& \text { where } i \in 0 \sim P-1, j \in 0 \sim Q-1
\end{aligned}
$$

Step 2) Binarization: After step 1, a threshold of $90 \%$ corresponds to the maximum pixel value of the scanned document is applied to separate the bright background and the dark halftone image. The binarized result $f^{(2)}(i, j)$ is shown in Fig. 7(b).

Step 3) Expansion and erosion: Since some background regions beyond the halftone image may be thresholded as black, we further apply morphological expansion and erosion operations to remove those unwanted areas. In general, the erosion number could be greater than the expansion number without affect the rotational angle. In detail, the reason of applying expansion is to remove some white dots in- 
side the halftone image, and the erosion is to remove the peripheral black regions. In order to make the overall procedure automatic, the expansion and erosion numbers are determined proportional to the overall black pixel of $f^{(2)}(i, j)$. According to numerous experiments, those parameters are fixed as

Expansion number $=0.045 \times \sqrt{\text { black pixel number, }}$

Erosion number $=0.295 \times \sqrt{\text { black pixel number }}$.

Herein, the processed result is denoted as $f^{(3)}(i, j)$.

Step 4) Edge detection: The following equation is applied to obtain the edge of the reserved area. The corresponding edge detected result is shown in Fig. 7(d). See (12), shown at the bottom of the page.

Step 5) Hough transform: Those pixels with value 255 in $f^{(4)}(i, j)$ are further transformed using Hough transform as below

$$
y=a x+b .
$$

Each pixel in $x-y$ domain corresponds to a line in $a-b$ domain as shown in Fig. 7(e). All the lines in a-b domain mostly intersect to four points, which correspond to two different slopes. The two points marked with red circles in the second quadrant correspond to the negative slope as indicated on the a-axis, where the value -15 is the pixel number apart from the origin in a-b domain, and the value 2.15 in the parentheses is the detected rotational angle. The intersected point 1 and point 1 correspond to the top and bottom edge of $f^{(4)}(i, j)$, respectively. Two other intersection points are located in the distance of the fourth quadrant and are not necessary to be included in the rotational angle detection. The gray area between point 1 and point 2 associates to the extended lines of the two intersection points in the fourth quadrant. In fact, the coordinates of point 1 and point 2 on the a axis are somewhat different. Hence, the rotational angle is defined as the average of the two values.

Step 6) Rotation correction: According to hundreds of testing with different print and scan resolution configurations, the proposed approach can detect the rotational angle correctly (comparing to the previous manual approach). Using the detected angle in step 5, the corresponding re-rotated results associate with $f^{(1)}(i, j)$ and $f^{(2)}(i, j)$ are shown in Fig. 7(f) and (g), which are denoted as $f^{(5)}(i, j)$ and $f^{(6)}(i, j)$. Note that, the origin of the re-rotation is not at the center of the whole scanned document, but in the center of the halftone image. According to the projected boundaries depicted in Fig. 7(f), where Fig. 7(f) and (c) are identical, the center coordinate is defined as $\left(\left(x_{\text {top }}+x_{\text {bottom }}\right) / 2,\left(y_{\text {left }}+y_{\text {right }}\right) / 2\right)$.

Step 7) Second round expansion and erosion: In this stage, it is to locate the whole area of the halftone image from the scanned document using the mask image as shown in Fig. 7(h). In addition to the original black noises beyond the halftone image in Fig. 7(b), some new black parts in the peripheries of the scanned document are involved after the re-rotation process in Fig. 7(h). As a result, the erosion number should be greater than in Step 4, and the expansion number can be kept equal as in Step 4. Based on numerous experiments, the second round expansion and erosion numbers defined below are selected.

2nd Expansion number

$$
\begin{aligned}
& =0.045 \times \sqrt{\text { black pixel number }} \\
& \text { 2nd Erosion number } \\
& =0.52 \times \sqrt{\text { black pixel number }}
\end{aligned}
$$

Apparently, as long as the erosion number is greater than expansion number, the reserved black region is shrunken. Hence, the second-time expansion should be conducted to recover the original area of the halftone image. The second-time expansion number is defined as

Second - time Erosion number

$$
=2 \text { ndErosion number }- \text { 2nd Expansion number. }
$$

The corresponding halftone region mask is denoted as $f^{(7)}(i, j)$ and is shown in Fig. 7(i).

Step 8) Image size normalization and binarization: The halftone image in $f^{(5)}(i, j)$ is extracted using the mask image $f^{(7)}(i, j)$. In general, the size of the print-and-scan halftone image is larger than the original size (according to hundreds of experiments), the preserved result is divided into $P \times Q$ square blocks (assume the original image is of size $P \times Q$ ), and the average pixel value inside each block is calculated to achieve image $f^{(8)}(i, j)$ as shown in Fig. 7(j), which is the size-normalized result.

Step 9) Normalized image Binarization: The image $f^{(8)}(i, j)$ is thresholded to recover the original halftone image (0-black and 255-white). Since the laser printer has the inherent problem of dot-gain effect, herein the threshold was set to be lower than 128 to overcome the darkening effect. According to the equipments, Epson AcuLaser C2000 printer and Epson Photo 700 scanner, the value 100 is a good

$$
f^{(4)}(i, j)= \begin{cases}255, & \text { if }\left|f^{(3)}(i+1, j)+f^{(3)}(i, j+1)-f^{(3)}(i-1, j)-f^{(3)}(i, j-1)\right| \geq 255 \\ 0, & \text { otherwise }\end{cases}
$$




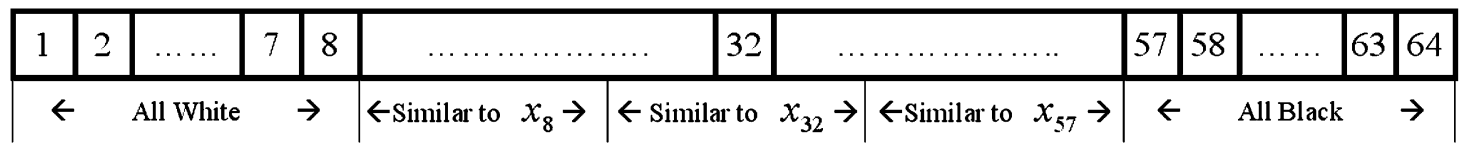

(a)

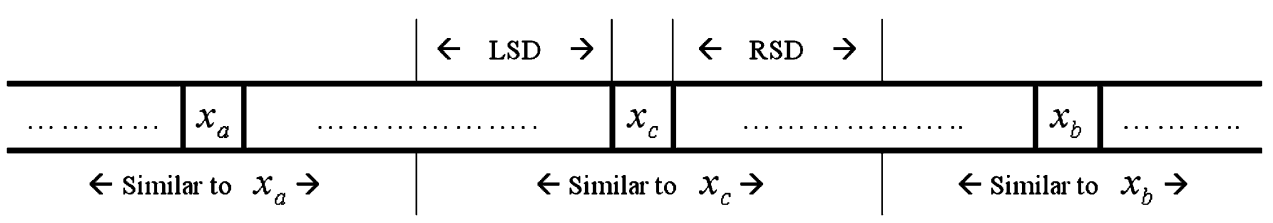

(b)

Fig. 8. One-dimensional representation of subimages. (a) Order of 64 subimages. (b) LSD and RSD of $x_{c}$.

choice. However, the value may slightly vary corresponding to different equipments. The threshold result is shown in Fig. 7(k).

Since the watermark and the embedded image are of the same pixel depth. The capacity in this work is defined as

$$
\text { capacity }=\frac{\text { size of watemark }}{\text { size of embedded image }} \text {. }
$$

The retrieved watermark may suffer from some error, yet the size remains unchanged. Hence, the capacity will not vary in practice according to the proposed algorithm.

In most applications, color halftone images are obtained by processing the three-color spaces (Red, Green, and Blue) separately. Based on this concept, we can embed the same watermark in these color spaces and retrieve it by majority voting. Documented in Section VI, the experiments demonstrate a high correct decoding rate.

One interesting question is whether it is possible to use a watermark based on the host image, so that the preprocessing to eliminate NIP can be avoid. In other words, the image quality can be maintained without degradation. Indeed, the scenario of using a dependent watermark may be utilized in authentication application. Where a dependent watermark is extracted from the host image via a corresponding hash function and then embedded. The decoded watermark can be used for verification. In this approach, the hash function key has to be taken as side information and sent to the decoder. Conversely, in many occasions, the watermarking is applied as ownership copyright protection. Hence, a totally image-independent watermark, such as the logo of a company or owner' digital signature, may be employed in this application. In these circumstances, it is not possible to always use a host image dependent watermark for embedding, and the proposed methods should be considered.

\section{Lossy Processing AND Progressive Coding}

\section{A. Progressive Coding}

A two-dimensional image such as Fig. 1(g) can be rearranged into the form of a one-dimension data sequence, as in Fig. 8(a). Subimages 1 to 8 are all white while subimages 57 to 64 are all black. Thus, the pixel values of these subimages need not be transmitted, because the receiver recognizes it when it receives an overhead bit stream of 64 bits. In this bit stream, the bit " 1 " appears in the interval $(1,32)$, which represents an all-white subimage. On the other hand, the bit " 1 " appears in the interval $(33,64)$, representing an all-black subimage. By doing so, we can reduce a quarter of data set in this example and the receiver can reconstruct it by filling all white (or black) in the relative positions of image after receiving this bit stream.

Fig. 1(g) shows that the subimages in position "32" or "33" preserve a significant portion of the original features. In other words, the subimages in position " 32 " or " 33 " should be transmitted and reconstructed first (in this paper we use 32). The receiver reconstructs the image by duplicating it and filling in positions 9 to 56, and then conduct inverse bit-interleaving.

The algorithm for the determination of the subsequent subimages to be transmitted is described as follows.

1) Let $x_{1}, x_{2}, \ldots \ldots, x_{m}$ represent the subimages shown in Fig. $8(\mathrm{a}) .\left(\mathrm{m}=n^{2}\right.$, here $\left.\mathrm{n}=8, \mathrm{~m}=64\right)$

2) Initialize flag $\left(x_{1}\right)=\operatorname{flag}\left(x_{2}\right)=\cdots=\operatorname{flag}\left(x_{m}\right)=0$.

3) $\operatorname{Set}$ flag $\left(x_{\text {upper }}\right)=\operatorname{flag}\left(x_{\text {lower }}\right)=1$. $\left(x_{\text {lower }}\right.$ represents the subimage before the subimage that a black pixel first appears if counting from $x_{1}$, where $x_{\text {upper }}$ represents the subimage before the subimage that the white pixel first appears if counting down from $x_{m}$. In the example, the $256 \times 256$ Lena image, $x_{\text {lower }}=x_{8}, x_{\text {upper }}=x_{57}$. When flag $(\bullet)=1$, means the subimage inside the parentheses should be transmitted or already sent)

4) Set flag $\left(x_{\text {middle }}\right)=1$, here $x_{\text {middle represents }} x_{32}$ in this paper.

5) Calculate the difference $D_{\mathrm{ab}}$ of every two adjacent subimages $x_{a}, x_{b}\left(x_{a}\right.$ and $x_{b}$ are already transmitted) such that $\operatorname{flag}\left(x_{a}\right)=\operatorname{flag}\left(x_{b}\right)=1$, define $D_{\mathrm{ab}}=D_{\mathrm{ab}}^{(1)}+D_{\mathrm{ab}}^{(2)}$, where

$$
\begin{aligned}
D_{\mathrm{ab}}^{(1)}= & \mathrm{H}\left(x_{a}, x_{a+1}\right)+\mathrm{H}\left(x_{a}, x_{a+2}\right)+\cdots \\
& +\mathrm{H}\left(x_{a}, x_{c}\right) \\
D_{\mathrm{ab}}^{(2)}= & \mathrm{H}\left(x_{c+1}, x_{b}\right)+\mathrm{H}\left(x_{c+2}, x_{b}\right)+\cdots \\
& +\mathrm{H}\left(x_{b-1}, x_{b}\right), \quad \mathrm{a} \leq \mathrm{c} \leq b
\end{aligned}
$$

$D_{\mathrm{ab}}^{(1)}, D_{\mathrm{ab}}^{(2)}$ satisfy the condition $D_{\mathrm{ab}}^{(1)} \cong D_{\mathrm{ab}}^{(2)}(\cong$ means as closer as possible, and the equation shown at the bottom of the page represents the weighted square distance between $x_{a}$ and $x_{a+1}$, where $P^{\prime} \times Q^{\prime}$ stands for the dimension of a subimage, and $w_{m, n}$ is the LMS filter described in Section III). Suppose $D_{\mathrm{ab}}^{\max }$ is the maximum difference of all the adjacent subimage pairs that $f \operatorname{lag}\left(x_{a}\right)=f \operatorname{lag}\left(x_{b}\right)=$ 


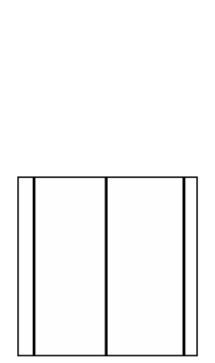

(a)

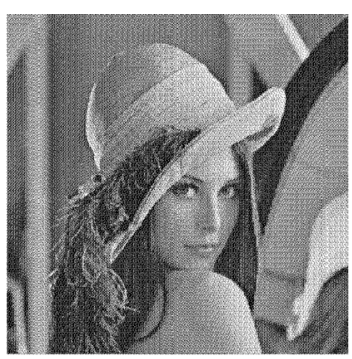

(b)

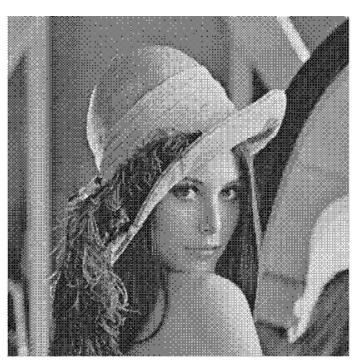

(c)

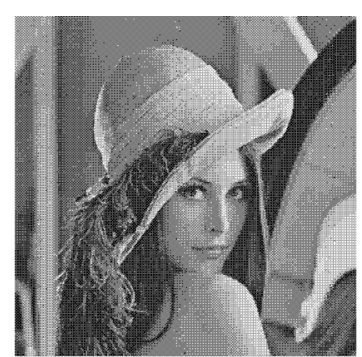

(d)

Fig. 9. Quality comparison. (a) Watermark of size $64 \times 64$ (b) Embedded dithered image with Hagit's [6] method, PSNR $=30.59$ dB. (c) Embedded dithered image with Fu and Au's [8] method, PSNR $=32.2 \mathrm{~dB}$. (d) Embedded dithered image with PSMOD, PSNR $=31.84 \mathrm{~dB}$. (d).

1 , then $x_{c}$ is the next subimage to be transmitted, and set flag $\left(x_{c}\right)=1$.

6) Fig. 8(b) shows the reconstructed left-side distance (LSD) and right-side distance (RSD) in the receiver, which satisfy the following conditions:

$$
\begin{aligned}
& \mathrm{H}\left(x_{a}, x_{a+1}\right)+\mathrm{H}\left(x_{a}, x_{a+2}\right)+\cdots+\mathrm{H}\left(x_{a}, x_{c-\mathrm{LSD}-1}\right) \\
& \cong \mathrm{H}\left(x_{c-\mathrm{LSD}}, x_{c}\right)+\mathrm{H}\left(x_{c-\mathrm{LSD}+1}, x_{c}\right)+\cdots \\
& \quad+\mathrm{H}\left(x_{c-1}, x_{c}\right) \\
& \mathrm{H}\left(x_{c}, x_{c+1}\right)+\mathrm{H}\left(x_{c}, x_{c+2}\right)+\cdots+H\left(x_{c}, x_{c+\mathrm{RSD}}\right) \\
& \cong \mathrm{H}\left(x_{c+\mathrm{RSD}+1}, x_{b}\right)+\mathrm{H}\left(x_{c+\mathrm{RSD}+2}, x_{b}\right)+\cdots \\
& \quad+\mathrm{H}\left(x_{b-1}, x_{b}\right)
\end{aligned}
$$

LSD and RSD are then represented by five bits and transmitted to the receiver as side information for reconstruction. In the receiver, the subimages covered by LSD and RSD should be placed the same values as subimage $x_{c}$.

\section{B. Lossy Processing and Entropy Coding}

In the previous section, we illustrate the procedure of progressive transmission for lossless reconstruction of the original dithered images. In some practical applications, perfect reconstruction may not be as important as the processing speed. Thus, it is of importance to analyze the trade-off between reconstruction errors and processing speed. Here in this section, we provide the overview of the algorithm for the improvement of processing speed with a lossy process, as follows.

1) Define a threshold $N_{\text {th }}$, as the total number of pixels of which the values can be changed in a reconstructed progressive dithered image.

2) Find the minimum minor pixel (minor pixel means black pixel in $x_{1} \sim x_{\text {middle }}$ or white pixel in $x_{\text {middle+1 }} \sim x_{m}$ ) number of the subimage simultaneously searching up from $x_{1}$ and down from $x_{64}$.

3) Reverse a minor pixel value (white to black, or black to white) of the subimage with minimum minor pixel as given in Step 2 and then subtract 1 from $N_{\text {th }}$.

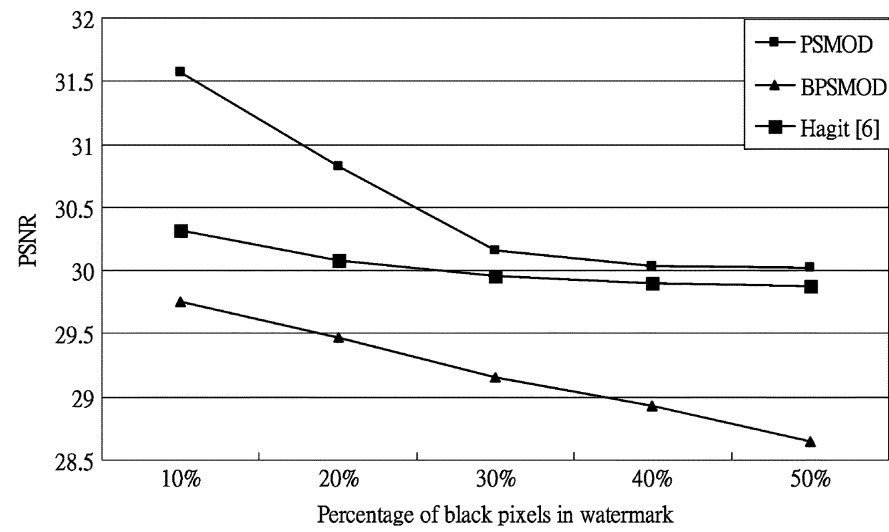

Fig. 10. PSNR versus percentage of black pixels in watermark.

4) Repeat Steps 2 and 3 until $N_{\text {th }}=0$ (when $N_{\text {th }}=0$ means there are already $N_{\text {th }}$ pixels changed in a dithered image).

Thus we can produce a bit-interleaved image with more all black (white) subimages, or generally speaking, an image with lower entropy.

Finally, we employ a lossless Huffman coder to perform further entropy coding of subimages to be transmitted. Because the pixels are average white in the lower half plane and black in the upper half plane of the bit-interleaved image, as shown in Fig. 1(g), the coding gain can be improved if we separate the Huffman coding process into the lower plane and upper plane.

\section{EXPERIMENTAL RESULTS}

To verify and document the performance of the proposed watermarking techniques, we conducted a sequence of experiments. In Fig. 9(a), we try to use three evident strips to demonstrate the quality degradation from the subjective evaluation. It is clear that, with Hagit's method [6], the embedded image shown in Fig. 9(b) has three traceable false contours in relative positions of watermark. However, it does not exist in Fig. 9(c) (Fu and Au's method [8]) and (d) (PSMOD). The error criterion, derived in Section III, is used for objective comparison.

$$
\mathrm{H}\left(x_{a}, x_{a+1}\right)=\sum_{i=1}^{P^{\prime}} \sum_{j=1}^{Q^{\prime}}\left\{\sum_{m, n \in R} \sum w_{m, n}\left[x_{a}(i+m, j+n)-x_{a+1}(i+m, j+n)\right]\right\}^{2}
$$




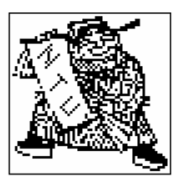

(a)

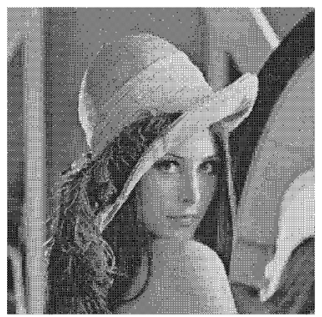

(c)

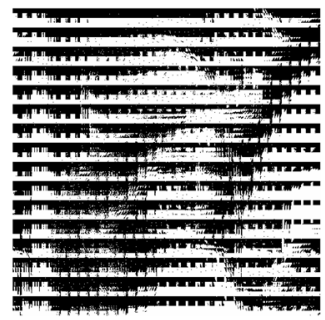

(d)

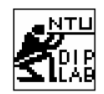

(b)

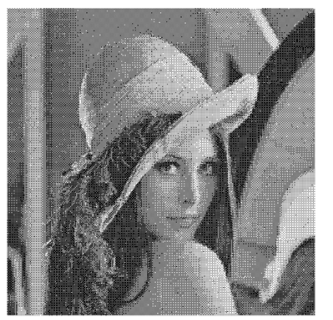

(e)

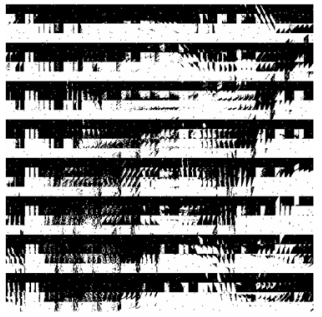

(f)

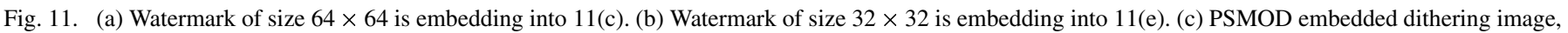

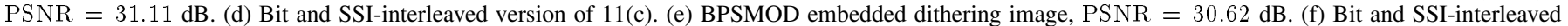
version of 11(e).

The PSNR are 30.59, $32.2 \mathrm{~dB}$, and $31.84 \mathrm{~dB}$ in Fig. 9(b), (c), and (d), respectively. According to the experiment results, the quality performance order, from the worst to the best, is Hagit's method, proposed method, and then Fu and Au's method. However, robustness was not taken into consideration in Fu and Au's method. Hence, the proposed method has the potential advantage in this regard.

The black pixels in watermark can cause the insertion of additional pseudo-pixels or SSI swapping, and consequently degradation to the quality of the embedded image. Fig. 10 illustrates the PSNR as functions of black-pixel percentage in watermark with pseudo-random generated black pixels. When the percentage exceeds $50 \%$, we can modify the algorithms by adding pseudo pixels or SSI swapping once if white pixels appear in watermark. The proposed PSMOD technique, even in the critical situation of $50 \%$ black pixels, can still achieve higher PSNR than Hagit's method. However, because the BPSMOD method needs to add pseudo pixels each time when NIP occurs, even though the watermark with all white pixels, the BPSMOD has the worst performance in PSNR. As discussed in Section IV-A, with the same halftone screen, the amount of NIP decreases with less further dividing in subimage. So in the following experiments we embed watermark of size $64 \times 64$ with PSMOD, and $32 \times 32$ with BPSMOD.

Fig. 11(a) and (b) show the original embedded watermarks of sizes $64 \times 64$ and $32 \times 32$, respectively. Fig. 11(a) is embedded into Fig. 11(c), and Fig. 11(b) is embedded into Fig. 11(e). Fig. 11(c) and (d) are the embedded dithering image and its SSI-interleaved result with the PSMOD technique. In Fig. 11(c) each subimage is divided into 512 SSI's (to 16 portions horizontally and to 32 portions vertically). Fig. 11(e) and (f) are the embedded dithered image and its SSI-interleaved result with the BPSMOD technique. The objective qualities of Fig. 11(c) and (e) are 31.11 and $30.62 \mathrm{~dB}$, respectively.

The BPSMOD can extract something even though the received halftone image in fact does not contain any embedded information. Hence, the BPSMOD is not applicable in copyright protection, since anyone can claim the extracted "thing" as their digital code. For this, the main application of BPSMOD is in secret communication. Suppose a decoded watermark is extracted

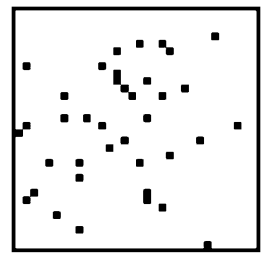

Fig. 12. Decoded meaningless result from Fig. 1(d).

from a non-embedded image, the extraction cannot deliver any information. Hence, the BPSMOD is feasible in this case. To prove this explanation, the watermark extracted from Fig. 1(d), which is not embedded any watermark beforehand, is shown in Fig. 12. As indicated above, the decoded result does not show any information of significance.

Subsequently, we conducted a series of experiments to demonstrate the robustness of these techniques. In Fig. 13, we analyzed the degradation of the embedded dithered as shown in Fig. 11(c) and (e) due to the cropping and tampering attacks. The correct decoding rates of $95.01 \%$ and $91.11 \%$ are obtained with cropping attack, and $94.62 \%$ and $90.32 \%$ are obtained with tampering attack. These decoded results are shown in Fig. 13(b), (d), (f), and (h), respectively.

Table II shows the average correct decoding rates of the embedded dithered images with 8 tested images as shown in Fig. 2, in various configurations, including $\mathrm{B} \& \mathrm{~W}$ and color images, as well as bitmap and scanned-and-printed images in different resolutions. Table II shows the color images, using majority voting as described in Section IV-C, result in greater accuracy than the $\mathrm{B} \& \mathrm{~W}$ images. The print-and-scan process reproduces the embedded image at $150 \mathrm{dpi}$ and scans at 150,450 , and 750 , respectively. The results also show that higher scan resolution improves the accuracy of the decoding rates.

The robustness of the proposed techniques is supported by two key features. First, based on experiments, after the pseudopixel insertion, $x_{i, j, k+1}^{\prime}$ always has one or more black pixels than $x_{i, j, k}^{\prime}$. Statistically the pair of SSI is unlikely to change to an NIP after distortions. In addition, the most common distortions are "clustered," such as cropping, tampering, as well as 


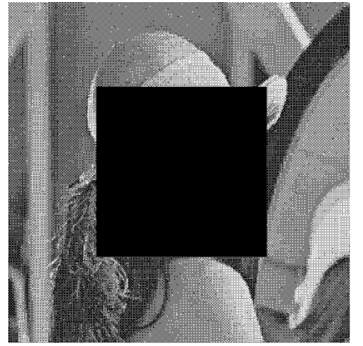

(a)

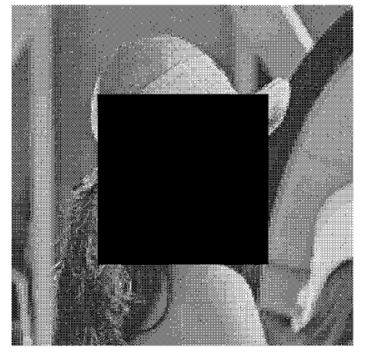

(e)

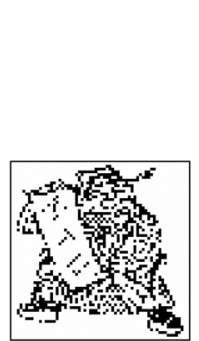

(b)

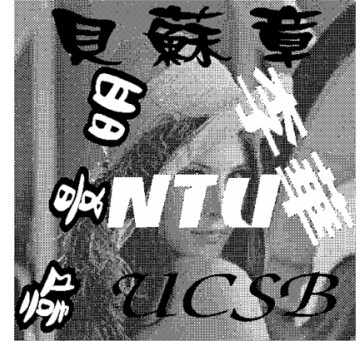

(c)

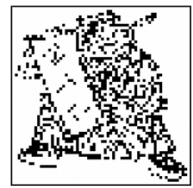

(d)

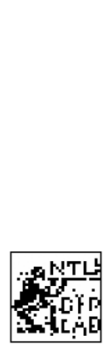

(f)

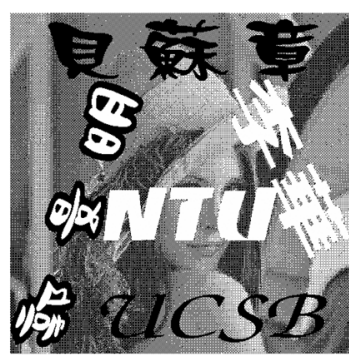

(g)

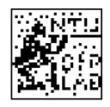

(h)

Fig. 13. Robust watermarking testing. (b), (d), (f), and (h) are decoded watermarks from (a), (c), (e), and (g) with correct decoding rates of 95.01, 94.62, 91.11, and $90.32 \%$, respectively. (a) and (e) are cropped by $1 / 4$. (c) and (g) are tampered.

TABLE II

Average CoRrect DECODED RATES

\begin{tabular}{|c|c|c|c|c|c|}
\hline \multicolumn{2}{|c|}{} & \multirow{2}{*}{ Bitmap } & \multicolumn{3}{c|}{ Print-and-scan } \\
\cline { 4 - 6 } \multicolumn{2}{|c|}{} & & $150 \mathrm{dpi}$ & $450 \mathrm{dpi}$ & $750 \mathrm{dpi}$ \\
\hline \multirow{2}{*}{ PSMOD } & B\&W & 100 & 89.23 & 91.74 & 92.06 \\
\cline { 2 - 6 } & Color & 100 & 91.45 & 93.87 & 94.19 \\
\hline \multirow{2}{*}{ BPSMOD } & B\&W & 100 & 68.12 & 74.17 & 80.34 \\
\cline { 2 - 6 } & Color & 100 & 75.31 & 84.35 & 93.01 \\
\hline
\end{tabular}

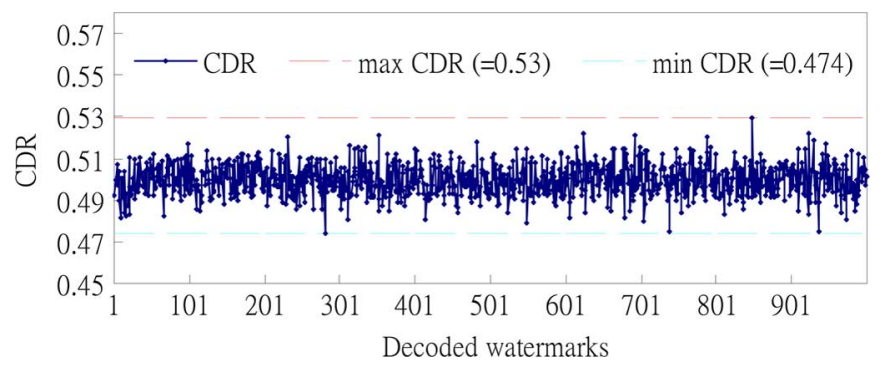

Fig. 14. False positive testing for the proposed PSMOD.

print-and-scan. This suggests that, after this kind of distortions, the two pixels within the same halftone screen region, corresponding to a pair of SSI, will change to black or white simultaneously. In most situations, the two pixels are of the same value (black or white) before distortion as shown in Fig. 1(f) and (h).

Some issues of the secure communication application in BPSMOD include capacity, imperceptible transparency, and statistical security. In capacity, the flexibility feature and the corresponding erroneous rate versus embedded amount are extensively discussed in Table I which associates to the discussions in Section IV-A and IV-B. The imperceptible transparency is also demonstrated in the bottom of Table I. The reason that the Table I is related to BPSMOD is because that all the NIPs in divided block or subblocks pairs are compensated by pseudo pixels, which is the important preprocessing of the BPSMOD. According to experiment results, a PSNR higher than $30 \mathrm{~dB}$ meets the imperceptible transparency requirement. Finally, the security of the BPSMOD relies on two stages, the first one is the algorithm itself, and the other one is the pseudo-random permutation key as indicated in Fig. 6. Summarizing the discussions given above, the proposed BPSMOD is qualified as a secure communication application.

Another issue is the false positive detection. In this work, the PSMOD require the original watermark to prove the ownership of the embedded image. On the other hand, the decoded result of BPSMOD should be meaningful to convey information to destination. In general, an unwatermarked image is difficult to extract a registered watermark or meaningful pattern. For this, we have tested 250 unwatermarked images, after printing at $150 \mathrm{dpi}$ and scanned at 150, 300, 450, and 750 different resolutions, respectively. Herein, the proposed resynchronization approach is applied. According to the experiment results, the correct decoding rates $(\mathrm{CDR})$ of those decoded watermarks with the registered watermark are totally irrelevant as shown in Fig. 14, where 1-250, 250-500, 501-750, and 751-100 associate to the CDR of watermarks decoded from scanned resolution 150, 300, 450, and 750, respectively. The maximum and minimum CDR are 0.53 and 0.474 , respectively. In addition, none of the decoded results are meaningful. Hence, it proves that the false positive result is nearly impossible happened in practical applications.

In progressive dithered halftone image coding, a standard $256 \times 256$ pixel 8-bits gray-tone image of Lena, as shown in Fig. 15(a), is used as the test image of the experiments. The results of progressive reconstruction are shown in Fig. 15(b)-(h), corresponding to step 1 to step 7 , which are the reconstructions from 1, 2, 4, 8, 16, 32, and 48 subimages. Fig. 15(h) is the final reconstruction from all 48 subimages. This is because there are 16 all black and all white subimages and only a bit stream will be required to transmit to represent the images.

The images of Peppers, Mandrill, Milk, and Airplane were also used for the experiments for comparison purposes, and the experimental PSNR corresponding to the number of steps is 


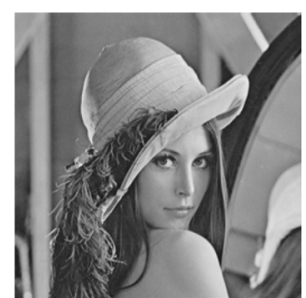

(a)

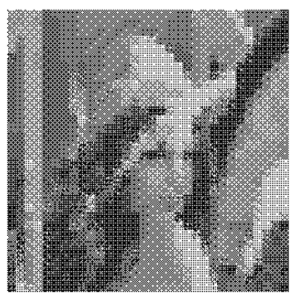

(e)

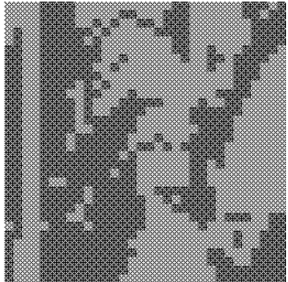

(b)

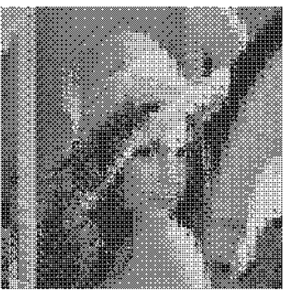

(f)

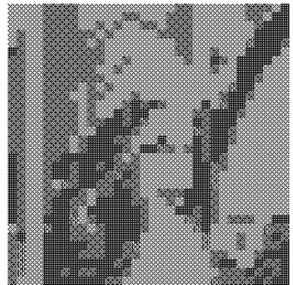

(c)

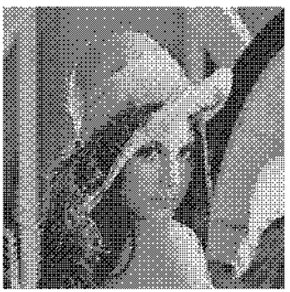

(g)

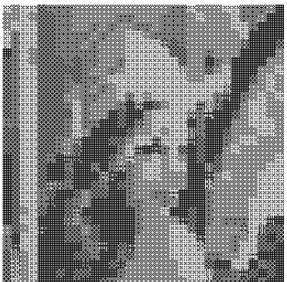

(d)

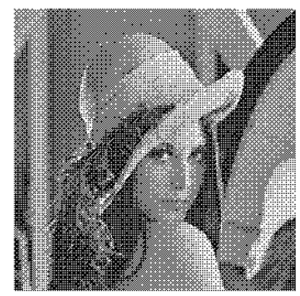

(h)

Fig. 15. (a) Original $256 \times 256$ gray-scaled Lena image. (b)-(h) Reconstructed dithering $256 \times 256$ Lena images in 7 steps (printed at 150 dpi).

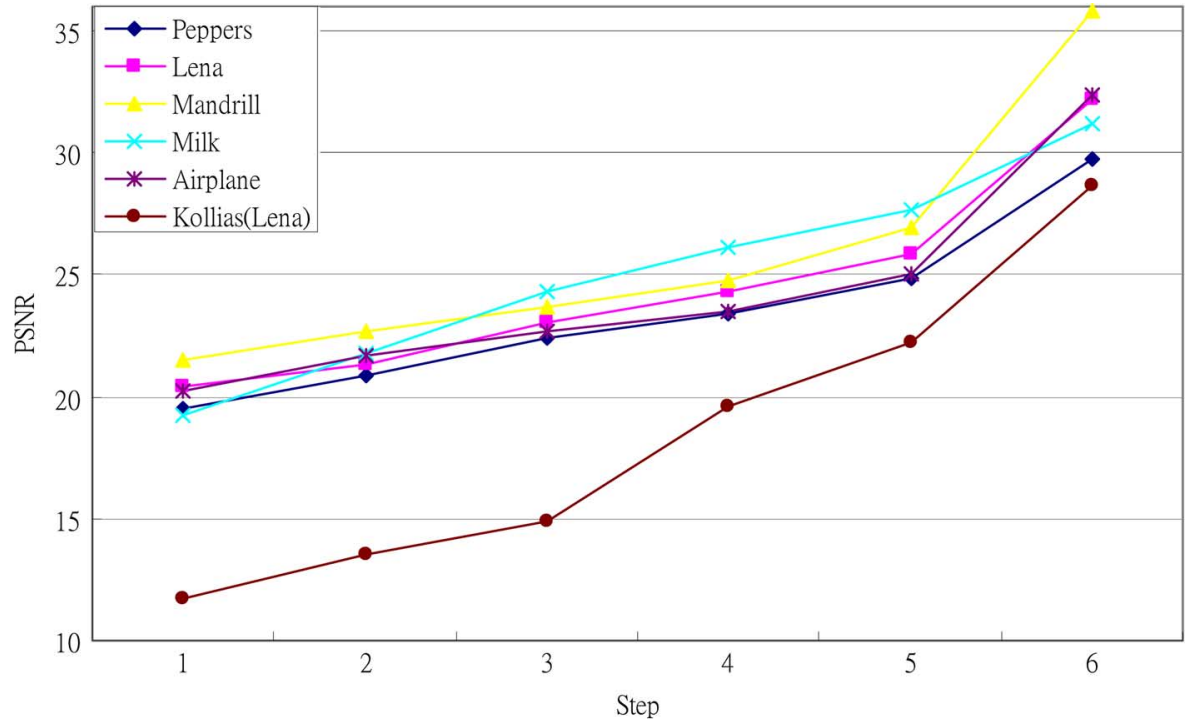

Fig. 16. PSNR versus reconstructed steps of five tested images.

TABLE III

Bit Rate With Lossless HufFMAn CODING

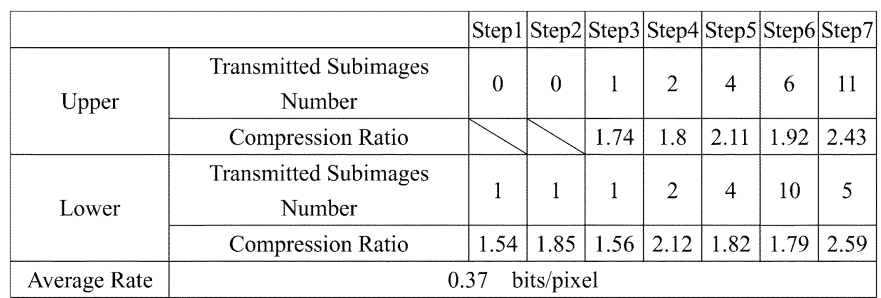

shown in Fig. 16. Since the image is completely reconstructed step 7, here we evaluate the reconstructed quality in each step by comparing with step 7 . Both images are halftone images, so
TABLE IV

BIT RATE OF Four Dithered IMAGES AfTER ENTROPy CODING

\begin{tabular}{|c|c|}
\hline Testing Images & Bit Rate (bits/pixel) \\
\hline Mandrill $\left(\mathrm{x}_{\text {lower }}=\mathrm{x}_{6}, \mathrm{x}_{\text {upper }}=\mathrm{x}_{53}\right)$ & 0.31 \\
\hline Peppers $\quad\left(\mathrm{x}_{\text {lower }}=\mathrm{x}_{1}, \mathrm{x}_{\text {upper }}=\mathrm{x}_{57}\right)$ & 0.39 \\
\hline Airplane $\left(\mathrm{x}_{\text {lower }}=\mathrm{x}_{5}, \mathrm{x}_{\text {upper }}=\mathrm{x}_{58}\right)$ & 0.37 \\
\hline Milk $\quad\left(\mathrm{x}_{\text {lower }}=\mathrm{x}_{2}, \mathrm{x}_{\text {upper }}=\mathrm{x}_{62}\right)$ & 0.3 \\
\hline
\end{tabular}

they are processed with the LMS filter described in Section II and as noted in denominator of modified PSNR as shown in (18) at the bottom of the page.

$$
\mathrm{PSNR}=10 \log _{10} \frac{P \times Q \times 255^{2}}{\sum_{i=1}^{P} \sum_{j=1}^{Q}\left[\sum_{u, v \in R} \sum w_{u, v}\left(b_{i+u, j+v}^{\text {step }}-b_{i+u, j+v}^{\text {step } x}\right)\right]^{2}}, \quad \text { where } x=1 \sim 6
$$


TABLE V

BIT RATE AND PSNR AFTER LOSSY PROCESS AND ENTROPY CODING

\begin{tabular}{|c|c|c|c|c|}
\hline \multirow[b]{2}{*}{ PSNR / Bit Rate } & \multicolumn{4}{|c|}{ Pixel Loss Number } \\
\hline & $\begin{array}{c}0 \\
\left(\mathrm{x}_{\text {lower }}=\mathrm{x}_{8}, \mathrm{x}_{\text {upper }}=\mathrm{x}_{57}\right)\end{array}$ & $\begin{array}{c}400 \\
\left(\mathrm{x}_{\text {lower }}=\mathrm{x}_{12}, \mathrm{X}_{\text {upper }}=\mathrm{x}_{50}\right)\end{array}$ & $\begin{array}{c}800 \\
\left(\mathrm{x}_{\text {lower }}=\mathrm{x}_{13}, \mathrm{x}_{\text {upper }}=\mathrm{x}_{47}\right)\end{array}$ & $\begin{array}{c}1200 \\
\left(\mathrm{x}_{\text {lower }}=\mathrm{x}_{14}, \mathrm{x}_{\text {upper }}=\mathrm{x}_{45}\right)\end{array}$ \\
\hline PSNR (dB) & & 22.14 & 19.13 & 17.37 \\
\hline Bit Rate & 0.37 & 0.3 & 0.29 & 0.26 \\
\hline
\end{tabular}

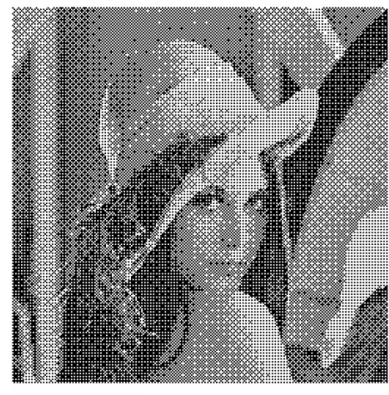

(a)

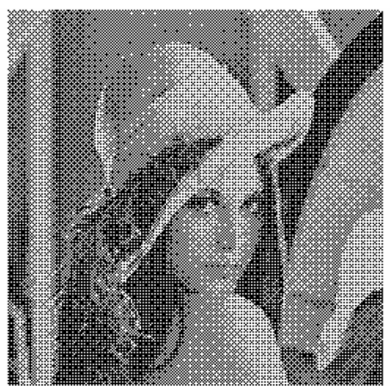

(b)

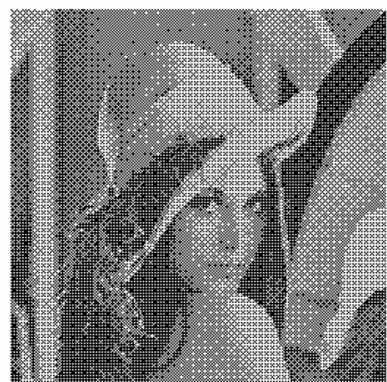

(c)

Fig. 17. Lossy progressive coding results with (a) 400 pixels' loss (PSNR $=30.93 \mathrm{~dB}$ ). (b) 800 pixels' loss (PSNR $=29.11 \mathrm{~dB})$. (c) 1200 pixels' loss $($ PSNR $=28.21 \mathrm{~dB})$.

The variable $\mathbf{b}_{\bullet}^{\text {step } x}$ stands for the reconstructed dithering image in step $\mathrm{x}$. The quality of the reconstructions showed noticeable improvement over the resultant images by the technique proposed by Kollias [21].

Table III shows the Huffman lossless compression ratio, and the occurrence number of the upper or lower subimages in each coding step with a $256 \times 256$ Lena test image. After completing the reconstruction, the average bit rate of the experiment is 0.37 bits/pixel.

Another set of four images was also applied for testing. Experimental results, documented in Table IV, show an average bit rate of 0.34 , compared to the resultant bit rate of 0.75 by Kollias [21]. Generally speaking, due to the high frequency components inherent in Mandrill, the compression ratio is more difficult to improve with traditional compression methods. Yet, this technique also achieved a low bit rate for the Mandrill image. This is because there are many all-black and all-white bit-interleaved subimages in the Mandrill image, which results in bit-rate reduction. This indicates that the bit rate of the proposed coding scheme is related to not only the complexity, but also the dynamic range concentration of the images.

Table V illustrates the experimental bit rates and relative PSNR of the progressive lossy coding of the Lena image described in Section V-B. The experimental results are documented in Fig. 17 for comparison. Fig. 17(a)-(c) are corresponding to $400,800,1200$ pixels' loss, respectively, at 150 dpi.

\section{CONCLUSION}

In this paper, two novel watermarking schemes, namely PSMOD and BPSMOD, for dithered images were presented. Both techniques demonstrated good recovery abilities in cropping, tampering, and print-and-scan distortions. In addition, it has been extended to color images and decoded, based on the concept of majority voting, and retained a high decoding rate. The watermarking schemes also provided satisfactory embedded quality and flexibility to situations of various embedded capacities. Although the technical discussions in this paper were limited to dispersed-dot dithering images, with slight modifications, it can also be generalized to clustered-dot dithering images in a similar manner.

Although the proposed technique PSMOD produce higher embedded quality and flexibility in handling different embedded capacities, the correct-decode rate is not as high as that achieved by the Hagit's method [6].

A new progressive coding algorithm for dithered images was also presented. The experimental results showed high-quality reconstruction of dithered images. In addition, the lossy and entropy coding procedure preserved the image quality at low coding rates. In this paper, the algorithm was applied to dispersed-dot dithering images. Nonetheless, it can be modified and generalized to include clustered-dot dithering images as well.

\section{REFERENCES}

[1] R. Ulichney, Digital Halftoning. Cambridge, MA: MIT Press, 1987.

[2] D. L. Lau and G. R. Arce, Modern Digital Halftoning. New York: Marcel Dekker, 2001.

[3] J. F. Jarvis, C. N. Judice, and W. H. Ninke, "A survey of techniques for the display of continuous-tone pictures on bilevel displays," Comp. Graph. Image Process., vol. 5, pp. 13-40, 1976.

[4] P. Stucki, MECCA-A multiple-error correcting computation algorithm for bilevel image hardcopy reproduction Zurich, Switzerland, Res. Rep. RZ1060, IBM Res. Lab., 1981.

[5] R. W. Floyd and L. Steinberg, "An adaptive algorithm for spatial gray scale," in Proc. SID 75 Dig. Soc. For Inf. Display, 1975, pp. 36-37.

[6] H. Z. Hel-Or, "Watermarking and copyright labeling of printed images," J. Electron. Imag., vol. 10, no. 3, pp. 794-803, Jul. 2001. 
[7] Z. Baharav and D. Shaked, "Watermarking of dither halftoned images," in Proc. IS\&T/SPIE Conf. Security Watermarking Multimedia Content 3657, 1999, pp. 307-316.

[8] M. S. Fu and O.C. Au, "Data hiding watermarking for halftone image," IEEE Trans. Image Process., vol. 11, no. 4, pp. 477-484, Apr. 2002.

[9] D. Kacker and J. P. Allebach, "Joint halftoning and watermarking," IEEE Trans. Signal Process., vol. 51, no. 4, pp. 1054-1068, Apr. 2003.

[10] J. R. Goldschneider, E. A. Riskin, and P. W. Wong, "Embedded color error diffusion," in Proc. 3rd IEEE Int. Conf. Image Process., Lausanne, Switzerland, 1996, vol. 1, pp. 565-568.

[11] J. R. Goldschneider, E. A. Riskin, and P. W. Wong, "Embedded multilevel error diffusion," IEEE Trans. Image Process., vol. 6, no. 7, pp. 956-964, Jul. 1997.

[12] M. S. Fu and O. C. Au, "Hiding data in halftone image using modified data hiding error diffusion," Proc. SPIE, vol. 4067, pp. 1671-1680, 2000.

[13] Z. M. Lu, H. Luo, and J. S. Pan, "Reversible watermarking for error diffused halftone image using statistical features," Lecture Notes Comput. Science, LNCS 4283, pp. 71-81, 2006.

[14] S. G. Wang and K. T. Knox, "Embedding digital watermarks in halftone screens," Proc. SPIE, vol. 3971, pp. 218-227, 2000.

[15] K. T. Knox, "Digital Watermarking Using Stochastic Screen Patterns," U.S. Patent 5734752.

[16] M. S. Fu and O. C. Au, "Data hiding in halftone images by stochastic error diffusion," in Proc. IEEE Int. Conf. Acoustics, Speech Signal Process., May 2000.

[17] M. S. Fu and O. C. Au, "Steganography in halftone images: Conjugate error diffusion," Signal Process., vol. 83, no. 10, pp. 2171-2178, Oct. 2003.

[18] M. S. Fu and O. C. Au, "Watermarking technique for color halftone images," in Proc. IEEE Int. Conf. Acoustics, Speech Signal Process., 2004, vol. 3, pp. III381-III384.

[19] S. C. Pei and J. M. Guo, "Data hiding in halftone images with noisebalanced error diffusion," IEEE Signal Process. Lett., vol. 10, no. 12, pp. 349-351, Dec. 2003.

[20] S. C. Pei and J. M. Guo, "Hybrid pixel-based data hiding and blockbased watermarking for error-diffused halftone images," IEEE Trans. Circuits Syst. Video Technol., vol. 13, no. 8, pp. 867-884, Aug. 2003.

[21] S. Kollias and D. Anastassiou, "A progressive scheme for digital image halftoning, coding of halftones, and reconstruction," IEEE J. Sel. Areas Commun., vol. 10, no. 5, pp. 944-951, Jun. 1992.

[22] C. N. Judice, "Data reduction of dither coded images by bit interleaving," in Proc. Soc. Inform. Display, 1976, vol. IT-17, no. 2, pp. 91-97.

[23] J. Mannos and D. Sakrison, "The effects of a visual fidelity criterion on the encoding of images," IEEE Trans. Inform. Theory, vol. IT-20, pp. 526-536, 1974.

[24] D. Anastassiou, "Neural network based digital halftoning of images," presented at the IEEE Symp. Circ. Syst., Helsinki, Finland, Jun. 1988, unpublished.

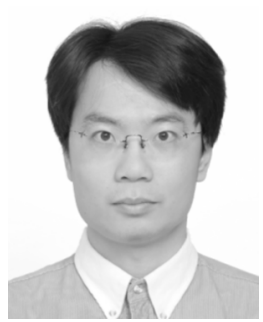

Jing-Ming Guo (M’06) was born in Kaohsiung, Taiwan, R.O.C., on November 19, 1972. He received the B.S.E.E. and M.S.E.E. degrees from National Central University, Taiwan, in 1995 and 1997, respectively, and the Ph.D. degree from the Institute of Communication Engineering, National Taiwan University, in 2004.

He was an Information Technique Officer in the Chinese Army from 1998 to 1999 . He is currently an Assistant Professor with the Department of Electrical Engineering, National Taiwan University of Science and Technology. He was granted the National Science Council scholarship for advanced research in the Department of Electrical and Computer Engineering,
University of California, Santa Barbara, from 2003 to 2004. His research interests include multimedia signal processing, multimedia security, digital halftoning, and digital watermarking.

Dr. Guo received the Acer Dragon Thesis Award in 2005, outstanding Paper Awards from IPPR, Computer Vision, and Graphic Image Processing in 2005 and 2006, and the Outstanding Faculty Award in 2002 and 2003. He is a member of IEICE and is a member of the Technical Committee of the IEEE Communications Society.

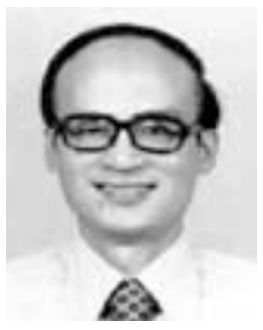

Soo-Chang Pei (M'86-SM'89-F'99) was born in Soo-Auo, Taiwan, R.O.C., in 1949. He received the B.S. degree from National Taiwan University in 1970 and the M.S. and Ph.D. degrees from the University of California, Santa Barbara, in 1972 and 1975, respectively, all in electrical engineering.

$\mathrm{He}$ was an Engineering Officer in the Chinese Navy Shipyard from 1970 to 1971 . From 1971 to 1975, he was a Research Assistant at the University of California, Santa Barbara. He was the Professor and Chairman in the Electrical Engineering Department, Tatung Institute of Technology and National Taiwan University, from 1981 to 1983 and 1995 to 1998 , respectively. Presently, he is the Professor in the Electrical Engineering Department at National Taiwan University. His research interests include digital signal processing, image processing, optical information processing, and laser holography.

Dr. Pei is a member of Eta Keppa Nu and the Optical Society of America. He received the National Sun Yet- Sen Academic Achievement Award in Engineering in 1984, the Distinguished Research Award from the National Science Council from 1990-1998, Outstanding Electrical Engineering Professor Award from the Chinese Institute of Electrical Engineering in 1998, and the Academic Achievement Award in Engineering from the Ministry of Education in 1998. He has been President of the Chinese Image Processing and Pattern Recognition Society in Taiwan from 1996-1998.

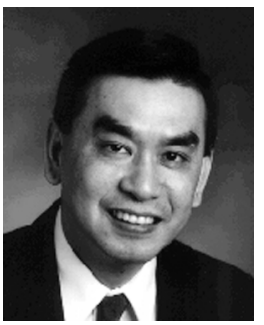

Hua Lee (F'92) received the Ph.D. degree from the University of California, Santa Barbara (UCSB), in 1980.

Prior to his return to UCSB in 1990, he was on the faculty of the University of Illinois at Urbana-Champaign. His research interests cover the areas of imaging system optimization, high-performance image formation algorithms, synthetic-aperture radar and sonar systems, acoustic microscopy, microwave nondestructive evaluation, and dynamic vision systems. His research laboratory was the first to produce the holographic and tomographic reconstructions from a scanning laser acoustic microscope, and his research team is also known as the leader in pulse-echo microwave nondestructive evaluation of civil structures and materials.

Dr. Lee is a Fellow of the Acoustical Society of America. In 1985, he received the Presidential Young Investigator Award from the National Science Foundation for his work in imaging system optimization. He was elected Professor of the Year in 1992 by the Mortar Board National Honor Society. He was also given the Nineteenth Pattern Recognition Society Award in 1993. He served as the Chairman of the 18th International Symposium on Acoustical Imaging in 1989 and the 13th International Workshop on Maximum Entropy and Bayesian Methods in 1993. From 1988 to 1994, he served as Editor of the International Journal of Imaging Systems and Technology. He also served as an Associate Editor of the IEEE TRANSACTIONS ON CIRCUITS AND SySTEMS FOR VIDEO TECHNOLOGY from 1992 to 1995, and Associate Editor of the IEEE TRANSACTIONS ON IMAGE PROCESSING from 1994 to 1996. 